\title{
Second order Galilean fluids and Stokes' law
}

\author{
Nabamita Banerjee, ${ }^{1, *}$ Sayali Atul Bhatkar, ${ }^{1, \dagger}$ and Akash Jain ${ }^{2, \$}$ \\ ${ }^{1}$ Dept. of Physics, Indian Institute of Science Education and Research (IISER), \\ Pune 411008, Maharashtra, India \\ ${ }^{2}$ Centre for Particle Theory and Dept. of Mathematical Sciences, Durham University, \\ Durham DH1 3LE, United Kingdom
}

(Received 25 January 2018; published 31 May 2018)

\begin{abstract}
We study the second derivative effects on the constitutive relations of an uncharged parity-even Galilean fluid using the null fluid framework. Null fluids are an equivalent representation of Galilean fluids in terms of a higher dimensional relativistic fluid, which makes the Galilean symmetries manifest and tractable. The analysis is based on the off-shell formalism of hydrodynamics. We use this formalism to work out a generic algorithm to obtain the constitutive relations of a Galilean fluid up to arbitrarily high derivative orders, and later specialize to second order. Finally, we study the Stokes' law which determines the drag force on an object moving through a fluid, in presence of certain second order terms. We identify the second order transport coefficients which leave the drag force invariant.
\end{abstract}

DOI: 10.1103/PhysRevD.97.096018

\section{INTRODUCTION}

Hydrodynamics is the universal low energy effective description of a finite temperature field theory around its thermodynamic equilibrium. A fluid configuration is described by certain fluid variables, typically chosen to be a normalized velocity, temperature, and chemical potentials associated with any internal symmetries. Dynamical equations for these variables are provided by the conservation of energy-momentum and any additional charges associated with the internal symmetries. By the virtue of being a low energy description, the length scales over which the fluid variables vary are taken to be large compared to the mean free path of the system. Thus, fluid energy-momentum tensor and charge currents admit a perturbative expansion in terms of derivatives of fluid variables, known as the fluid constitutive relations. At any given order in the derivative expansion, the constitutive relations contain all the possible tensor structures made out of fluid variables and their derivatives, multiplied with arbitrary functions of temperature and chemical potentials. These coefficients are known as the transport coefficients of the fluid. It is well known that if the dissipative hydrodynamics is truncated only at first derivative order with shear and bulk viscosity coefficients,

\footnotetext{
*nabamita@iiserpune.ac.in

† sayali.bhatkar@students.iiserpune.ac.in

akash.jain@durham.ac.uk
}

Published by the American Physical Society under the terms of the Creative Commons Attribution 4.0 International license. Further distribution of this work must maintain attribution to the author(s) and the published article's title, journal citation, and DOI. Funded by SCOAP ${ }^{3}$. there are always linearized fluctuations for which the wavefront speed is superluminal [1-3] and thus the causal structure of the theory is broken. Thus within the hydrodynamic framework, it was noted long ago by Muller, Israel, and Stewart [4-6] that one needs to go beyond the first derivative order and add specific second order terms to address the issue of causality. Since a causal system of second-order hydrodynamic equations is required in many situations, such as numerical simulations [7,8], a more systematic and detailed analysis of second order fluid transport has since been performed [9].

Although relativistic hydrodynamics is extremely useful at various physical fronts, nonrelativistic hydrodynamics also enjoys an active interest in the physics community. On our day to day energy scales, the world is governed by nonrelativistic physics. In this sense, a nonrelativistic fluid can be thought of as an effective version of a relativistic fluid under a nonrelativistic (large speed of light) limit. Hence, it is natural to expect that the associated constitutive relations, obtained as an effective description of a relativistic theory, may contain new terms which were not considered in the coarse grained description of relativistic hydrodynamics. This is apparent even at the first derivative order wherein the nonrelativistic fluid contains an additional transport coefficient called the thermal conductivity.

Conventionally, nonrelativistic hydrodynamics is studied by writing down a fluid theory whose fundamental symmetry group is Galilean as opposed to Poincaré for relativistic hydrodynamics. However, as the Galilean symmetry treats time and space coordinates on a different footing, the analysis becomes much more cumbersome. In a series of papers [10-13], we devised a new mechanism to study 
nonrelativistic hydrodynamics. We constructed a tweaked relativistic fluid in one higher dimension, called a null fluid, and showed that it is equivalent to a Galilean fluid in all respects. We also provided a simple dictionary, using the well-established procedure of null reduction [14], to translate between the null fluid and conventional Galilean fluid languages. Apart from representing Galilean fluids in a familiar and intuitive relativistic framework, null fluids also allow us to directly import the well developed results and machinery of relativistic hydrodynamics into Galilean fluids. For instance, we have successfully employed the null fluid framework to study first order (anomalous) charged Galilean hydrodynamics in [10,11], using both the second law of thermodynamics and equilibrium partition functions $[15,16]$ to determine physical constraints on the transport coefficients. We have also introduced the off-shell formalism for relativistic hydrodynamics [17] into Galilean hydrodynamics, and used it to study the influence of anomalies on the Galilean fluid constitutive relations [12] following the corresponding development in relativistic hydrodynamics (see [17-19] and references therein). Recently, following the analysis of relativistic superfluids in [20-22], we studied first order (anomalous) Galilean superfluids in [13]. These results were used to study surface transport in Galilean superfluids in [23].

In this paper, our aim is to perform a complete analysis of second order Galilean hydrodynamics using null fluids. As we stated above, causality demands the inclusion of second order transport coefficients in relativistic hydrodynamics, so it is natural to expect an impression of these to propagate into nonrelativistic hydrodynamics as well. Moreover, since null fluids are a relativistic system, we are also required to include the second order terms on grounds of consistency with causality. We focus on the parity-even uncharged case for computational simplicity, although the generalization of our results, albeit computationally involved, should be straight forward. We start by outlining a generic algorithm to work out the constitutive relations of a Galilean fluid up to arbitrary orders in derivative expansion in offshell formalism. This is based on a recent classification scheme for the entire relativistic hydrodynamic transport presented in $[22,24,25]$. Later, we use this algorithm to study the second order Galilean fluids. We find that in addition to the pressure at ideal order, and bulk viscosity, shear viscosity, and thermal conductivity at first order, there are 25 coefficients at second order. 5 of them are hydrostatic, i.e., they govern the equilibrium configuration of the fluid. Nine are dissipative, i.e., they are responsible for the production of entropy during dynamical processes, while the remaining 11 quantify dynamical processes which do not cause any dissipation.

To explore the physical significance of these second order transport coefficients, we study the effect of some representative terms on the Stokes' law. It is a famous hydrodynamic equation with numerous applications in physics and even in biology, that determines the drag force experienced by a body when moving through a fluid. If we acknowledge that there is an underlying causal relativistic theory behind our nonrelativistic fluid, of which our nonrelativistic fluid is just a low-energy description, this simple law will get modified by inclusion of the appropriate second order effects. In this article, we shall explore how some of the second order transport coefficients appearing in nonrelativistic hydrodynamics might affect the Stokes' law. We also identify a class of second order terms, which leave the Stokes' law invariant.

We should comment that in this paper we use the terms "nonrelativistic fluid" and "Galilean fluid" interchangeably. In principle, a Galilean fluid is defined as the most generic fluid which obeys Galilean symmetries. In this sense, a nonrelativistic fluid is a special kind of Galilean fluid which follows by taking a nonrelativistic limit of a relativistic system. This is to say that there might be some additional constraints on the Galilean fluid, following from the requirement that it should follow under a nonrelativistic limit. In our previous work however (see Sec. 5 of [13]), we argued that there are no such additional constraints and that every Galilean fluid can in fact be obtained via a nonrelativistic limit.

The paper is organized as follows. We start Sec. II with a brief review of null fluids and offshell formalism. We outline the generic algorithm to construct the null fluid constitutive relations at arbitrarily high derivative orders in Sec. II C, and illustrate how first order constitutive relations fit into this scheme in Sec. II D. In Sec. III, we use this algorithm to work out the second order constitutive relations of a Galilean fluid. For readers not interested in the computational details, the results have been summarized in Tables II-VI. In Sec. IV, we review the translation of null fluid constitutive relations into the conventional Galilean notation, and use it to obtain the constitutive relations of a Galilean fluid up to second derivative order. The final results gave been given in Tables VIII-X. We explore how these second order terms might affect the well known Stokes' law in Sec. V, exploring the physical significance of our results. Finally, we close the paper with some discussion in Sec. VI.

\section{CRASH COURSE IN NULL FLUIDS}

In [11] we proposed "null fluids" as a new viewpoint of Galilean fluids. The main benefit of working in this formalism is that it is effectively a "relativistic embedding" of a Galilean fluid into one higher dimension. This enables us to import the preexisting relativistic machinery and intuition into Galilean hydrodynamics. In this section we collect some of the results about null fluids which we use throughout this paper. The discussion is self contained, albeit brief. For more details, the reader is encouraged to refer to our previous papers, especially $[11,13,22]$. 


\section{A. Null backgrounds and null fluids}

Simply put, hydrodynamics is the study of long wavelength fluctuations of a quantum system on top of some slowly varying background fields. In this sense, the rules of hydrodynamics are governed by the background we start with. For example, consider a $(d+2)$-dimensional background with a metric $g_{M N}$ and an invariance under diffeomorphisms which act on the metric as

$$
\delta_{\mathcal{X}} g_{M N}=£_{\chi} g_{M N}=\nabla_{M} \chi_{N}+\nabla_{N} \chi_{M} .
$$

Here $\mathcal{X}=\left\{\chi^{M}\right\}$ are some arbitrary parameters and $\nabla_{M}$ is the covariant derivative associated with the Levi-Civita connection

$$
\Gamma_{M N}^{R}=\frac{1}{2} g^{R S}\left(\partial_{M} g_{N S}+\partial_{N} g_{M S}-\partial_{R} g_{M N}\right) .
$$

Hydrodynamics on this background corresponds to a usual (uncharged) relativistic fluid. To study null fluids however, we need to tweak this background by introduction of a vector field $\mathcal{V}=\left\{V^{M}\right\}$ which is null $V^{M} V_{M}=0$, covariantly constant $\nabla_{M} V^{N}=0$ and is an isometry

$$
\delta_{\mathcal{V}} g_{M N}=£_{V} g_{M N}=\nabla_{M} V_{M}+\nabla_{N} V_{M}=0 .
$$

We call these null backgrounds. They provide a natural "embedding" for Galilean (Newton-Cartan) backgrounds into a relativistic spacetime of one higher dimension. We will only be interested in fluctuations that respect the symmetries of the background, i.e., they must transform appropriately under diffeomorphisms and admit $\delta_{\mathcal{V}}=0$. A few key points to note about null backgrounds: the Riemann curvature tensor

$$
R^{M}{ }_{N R S}=2 \partial_{[R} \Gamma^{M}{ }_{S] N}+2 \Gamma^{M}{ }_{T[R} \Gamma^{T}{ }_{S] N},
$$

has vanishing contractions with $\mathcal{V}: R^{M}{ }_{N R S} V^{N}=$ $2 \nabla_{[R} \nabla_{S]} V^{M}=0$. Furthermore, we have a consistency condition on $\mathcal{V}: H_{M N} \equiv 2 \partial_{[M} V_{N]}=2 \nabla_{[M} V_{N]}=0$. From a mathematical standpoint, perhaps it is more natural to introduce a torsion tensor $\mathrm{T}^{R}{ }_{M N}$, in presence of which this condition becomes $V_{R} \mathrm{~T}^{R}{ }_{M N}=H_{M N}$ and lifts the constraint from $\mathcal{V}$. However, in interest of simplicity, we will work with torsionless null backgrounds and comment on the "unnaturalness" as it arises.

Given the diffeomorphism symmetry, Noether theorem implies that our theory has an energy-momentum tensor $T^{M N}$ in its spectrum. The respective conservation law is given as

$$
\nabla_{M} T^{M N}=0 .
$$

On null backgrounds we must further require that $\delta_{\mathcal{V}} T^{M N}=0$. Consequently, $T^{M N}$ is only defined up to terms proportional to $V^{M} V^{N}$ as $\nabla_{M}\left(\# V^{M} V^{N}\right)=V^{N} \delta_{\mathcal{V}} \#=0$.
We will extensively use this freedom to ignore terms proportional to $V^{M} V^{N}$ in $T^{M N}$ throughout this paper.

Having $(d+2)$ independent components, the conservation law (5) can provide dynamics for a "fluid theory" formulated in terms of arbitrary $(d+2)$ variables. We choose these to be a normalized null fluid velocity $u^{M}$ (with $u^{M} V_{M}=-1, u^{M} u_{M}=0$ ), a temperature $T$, and a mass chemical potential $\mu_{m}$, collectively known as the hydrodynamic fields. We sometimes work with a scaled mass chemical potential $\varsigma=\mu_{m} / T$. On a null background, we must further demand these fields to be compatible with $\mathcal{V}$, i.e., $\delta_{\mathcal{V}} u^{M}=\delta_{\mathcal{V}} T=\delta_{\mathcal{V}} \mu_{m}=0$. In general, $T^{M N}$ can arbitrarily depend on the hydrodynamic and background fields. But fortunately in hydrodynamics, we are only interested in low energy fluctuations of the hydrodynamic fields and a slowly varying background. This allows us to treat derivatives as a perturbation parameter. A null fluid is therefore completely characterized by a covariant expression for $T^{M N}$ in terms of $g_{M N}, V^{M}, u^{M}, T, \mu_{m}$ and their derivatives, truncated to a desired derivative order, known as the null fluid constitutive relations. At any given order, the constitutive relations can admit some tensor structures made out of the constituent fields and their derivatives called data, multiplied with arbitrary functions of $T$ and $\mu_{m}$ called transport coefficients. To this end, hydrodynamics is just a combinatorial exercise of enlisting all the possible tensor structures at a given order in derivatives.

To make things more interesting, we need to realize that fluids are thermodynamic systems. We have already imposed the first law of thermodynamics (conservation of energy) implicitly in form of the conservation laws (5). In addition, they are also required to satisfy a version of the second law of thermodynamics. It states that there must exist an entropy current $J_{S}^{M}$ whose divergence is positive semidefinite everywhere, i.e.,

$$
\nabla_{M} J_{S}^{M}=\Delta \geq 0,
$$

as long as the fluid is thermodynamically isolated [i.e., conservation laws Eq. (5) are satisfied]. Note that like $T^{M N}$, the entropy current $J_{S}^{M}$ is only defined up to terms proportional to $V^{M}$. In null hydrodynamics, our goal is to find the most generic constitutive relations $T^{M N}$ and some associated $J_{S}^{M}$ and $\Delta$ order by order in derivative expansion, such that Eq. (6) is satisfied for all thermodynamically isolated fluids. As innocuous as this statement sounds, the complexity involved drastically increases as we increase the derivative order. There is however, an equivalent but much neater way to work out these constitutive relations, called the off-shell formalism [17]. In the next subsection, we adapt this formalism to null fluids.

\section{B. Off-shell formalism}

As it turns out, most of the trouble while implementing Eq. (6) roots from the fact that it needs to only be imposed 
on the solutions of the equations of motion, i.e., onshell. We can relax this condition by coupling the fluid to an external momentum source $P_{\mathrm{ext}}^{M}$, so that the conservation law (5) is no longer satisfied. Having done that, Eq. (6) gets modified with an arbitrary combination of $P_{\mathrm{ext}}^{M}$ giving us

$\nabla_{M} J_{S}^{M}+\beta_{M} P_{\mathrm{ext}}^{M}=\nabla_{M} J_{S}^{M}+\beta_{M} \nabla_{M} T^{M N}=\Delta \geq 0$,

where $\mathcal{B}=\left\{\beta^{M}\right\}$ is an arbitrary vector multiplier. This equation is referred to as the off-shell second law of thermodynamics. It can be rewritten into a more useful form by defining a free energy current $G^{M}$

$$
-\frac{G^{M}}{T}=N^{M}=J_{S}^{M}+T^{M N} \beta_{N} .
$$

Equation (7) now turns into a conservation equation for free energy

$$
\nabla_{M} N^{M}=\frac{1}{2} T^{M N} \delta_{\mathcal{B}} g_{M N}+\Delta, \quad \Delta \geq 0 .
$$

Here $\delta_{\mathcal{B}} g_{M N}$ is of course $2 \nabla_{(M} \beta_{N)}$. Recall that the hydrodynamic fields $u^{M}, T$, and $\mu_{m}$ were some arbitrary fields chosen to describe the fluid. Like in any field theory, they are permitted to admit an arbitrary redefinition among themselves without changing the physics. This huge amount of freedom can be fixed by explicitly choosing

$$
\begin{aligned}
u^{M} & =-\frac{\beta^{M}}{V_{M} \beta^{M}}+\frac{\beta^{R} \beta_{R} V^{M}}{2\left(V_{N} \beta^{N}\right)^{2}}, \\
T & =-\frac{1}{V_{M} \beta^{M}}, \quad \mu_{m}=\frac{\beta^{M} \beta_{M}}{2\left(V_{N} \beta^{N}\right)^{2}},
\end{aligned}
$$

or conversely

$$
\beta^{M}=\frac{1}{T}\left(u^{M}-\mu_{m} V^{M}\right) .
$$

It follows that $\beta^{M}$ can be understood as a rewriting of the conventional hydrodynamic fields $u^{M}, T$ and $\mu_{m}$. Although this is a very convenient "frame" choice for our analysis, it might not be the most useful one for applications. But once we have obtained a consistent set of constitutive relations, we can always field transform to any desired frame.

To agree with the second law of thermodynamics, we need to find the most generic $T^{M N}$ written in terms of $\beta^{M}$, $g_{M N}$ and $V^{M}$ which satisfies Eq. (9) for some $N^{M}$ and $\Delta$. There is one minor subtlety to keep in mind though: $T^{M N}$ found this way will also contain information about the external sources $P_{\mathrm{ext}}^{N}=\nabla_{M} T^{M N}$. Therefore, in the end we must identify the constitutive relations which are related to each other up to combinations of equations of motion or their derivatives. Generically, equations of motion determine the "time" derivative of the fundamental fields, so without any loss of generality we can use them to eliminate $u^{M} \nabla_{M} \beta_{N}+u^{M} \nabla_{N} \beta_{M}=u^{M} \delta_{\mathcal{B}} g_{M N}$ from our constitutive relations. Consequently, we will only be interested in the constitutive relations $T^{M N}$ which are independent of $u^{M} \delta_{\mathcal{B}} g_{M N}$.

\section{Classification of constitutive relations}

We need to find the most generic solutions to Eq. (9) up to a given order in derivatives. We could take the "bruteforce" approach wherein we plug in the most generic expressions for $T^{M N}$ and $N^{M}$ up to a given derivative order, and find constraints arising from $\Delta \geq 0$. These constraints can be fairly complicated at higher derivative orders and crucially depend on the choice of basis for the tensordecomposition of $T^{M N}$ and $N^{M}$. But we can do better: we can write down a "solution generating algorithm" following [22,24] which will work at arbitrarily high derivative orders, as we now outline. We will then go on to apply this algorithm to second order null fluids in Sec. III. The discussion in this subsection parallels [22].

First and foremost we consider trivial solutions of Eq. (9) which do not contribute to the constitutive relations:

(i) Entropy transport (Class S): These are solutions of Eq. (9) of the kind $N^{M}=N_{\mathrm{S}}^{M}, T_{\mathrm{S}}^{M N}=0$ such that $\nabla_{M} N_{\mathrm{S}}^{M}=\Delta_{\mathrm{S}}$ is a quadratic form. They contain, for example, $N_{\mathrm{S}}^{M}=\nabla_{N} X^{[M N]}$ for an arbitrary antisymmetric tensor $X^{[M N]}$ or $N_{\mathrm{S}}^{M}=\mathcal{S} V^{M}$ for an arbitrary scalar $\mathcal{S}$, for both of which $\Delta_{\mathrm{S}}=0$. Examples for $\Delta_{\mathrm{S}} \neq 0$ are slightly complicated but can be obtained after some effort. These solutions correspond to the transport of entropy in a fluid $\left(J_{S}^{M}\right)_{\mathrm{S}}=N_{\mathrm{S}}^{M}$, without any transport of energy-momentum.

Note that if $T^{M N}$ is a solution to Eq. (9) for some free energy current $N^{M}$, then instead the free energy current $N^{M}+N_{\mathrm{S}}^{M}$ would also do. Therefore to satisfy the second law of thermodynamics for a given set of constitutive relations $T^{M N}$, we can choose any entropy current from the equivalence class $J_{S}^{M} \sim J_{S}^{M}+N_{S}^{M}$. In a strict sense therefore, Class S solutions are not really "hydrodynamic." They merely parametrize the multitude of entropy currents which might satisfy the second law for a given set of constitutive relations.

Getting these redundancies out of the way, we can now focus on the actual physical solutions. We broadly split the constitutive relations into two sectors: hydrostatic and nonhydrostatic based on the tensor structures that go into making them. Nonhydrostatic tensor structures are those which contain at least one instance of $\delta_{\mathcal{B}} g_{M N}$ or its derivatives. On the other hand, hydrostatic tensor structures are the largest collection of independent tensor structures with no nonhydrostatic linear combination. The terminology is based on the concept of equilibrium: a background is said to admit a hydrostatic configuration if it has a timelike isometry $\mathcal{K}=\left\{K^{M}\right\}$. On such a background, a hydrostatic 
configuration is given by $\beta^{M}=K^{M}$ which trivially satisfies the equations of motion. By definition therefore, hydrostatic constitutive relations are the only ones that survive in a hydrostatic configuration and govern the equilibrium physics.

Spoiler alert. The second law imposes strict constraints in the hydrostatic sector allowing for only specific combinations of tensor structures to appear, while in the nonhydrostatic sector it merely gives a few inequalities at the first order in derivatives and none thereafter [26,27].

(i) Hydrostatic transport $\left(\right.$ Classes $\mathrm{H}_{S}, \mathrm{H}_{V}$ and A): These are solutions of Eq. (9) made purely out of hydrostatic tensor structures. They have $\Delta=0$ and are completely characterized by a free energy current of the form

$$
N^{M}=\left(\mathcal{N} \beta^{M}+\Theta_{\mathcal{N}}^{M}\right)+\mathbb{N}^{M} .
$$

Here $\mathcal{N}$ is the most generic hydrostatic scalar, while $\Theta_{\mathcal{N}}^{M}$ is an appropriate nonhydrostatic vector added to ensure that the term in parenthesis has exactly one bare (without derivatives) $\delta_{\mathcal{B}} g_{M N}$ in its divergence to match-up with the right-hand side (RHS) of Eq. (9). We can work out the corresponding constitutive relations, called Class $\mathrm{H}_{S}$ for hydrostatic scalars, by noting that:

$$
\begin{aligned}
\nabla_{M}\left(\mathcal{N} \beta^{M}\right)= & \frac{1}{\sqrt{-g}} \delta_{\mathcal{B}}(\sqrt{-g} \mathcal{N}) \\
= & \frac{1}{2} \mathcal{N} g^{M N} \delta_{\mathcal{B}} g_{M N}+\frac{\partial \mathcal{N}}{\partial g_{M N}} \delta_{\mathcal{B}} g_{M N} \\
& +\frac{\partial \mathcal{N}}{\partial\left(\partial_{R} g_{M N}\right)} \partial_{R} \delta_{\mathcal{B}} g_{M N}+\cdots \\
\equiv & \frac{1}{2} T_{\mathrm{H}_{S}}^{M N} \delta_{\mathcal{B}} g_{M N}-\nabla_{M} \Theta_{\mathcal{N}}^{M} .
\end{aligned}
$$

Here $\nabla_{M} \Theta_{\mathcal{N}}^{M}$ is a total derivative term gained after successive differentiation by parts, which defines $\Theta_{\mathcal{N}}^{M}$. Note that if $\mathcal{N}$ has some total derivative terms, i.e., $\mathcal{N}=\nabla_{M} X^{M}$ for an arbitrary hydrostatic vector $X^{M}$, upon choosing $\Theta_{\mathcal{N}}^{M}=-\frac{1}{\sqrt{-g}} \delta_{\mathcal{B}}\left(\sqrt{-g} X^{M}\right)$ the free energy current $\mathcal{N} \beta^{M}+\Theta_{\mathcal{N}}^{M}=\beta^{M} \nabla_{N} X^{N}-$ $\frac{1}{\sqrt{-g}} \delta_{\mathcal{B}}\left(\sqrt{-g} X^{M}\right)=2 \nabla_{N}\left(\beta^{[M} X^{N]}\right)$ has zero divergence, and hence belongs to Class $\mathrm{S}$. Class $\mathrm{H}_{S}$ constitutive relations are therefore characterized by the most generic hydrostatic scalar $\mathcal{N}$ up to total derivatives.

On the other hand, $\mathbb{N}^{M}$ contains all the hydrostatic vectors transverse to $u^{M}$ and $V^{M}$ whose divergence contains exactly one bare $\delta_{\mathcal{B}} g_{M N}$. Intuitively, this means that we want to find "spatial" vectors without any "time" derivative in them, whose divergence however still contains a "time" derivative. Slight thought will reveal that this is only possible in parity-odd sector, which we are not considering in this work. For completeness, we should mention that they contain contributions from anomalies (Class A) and some other parity-odd terms commonly dubbed as transcendental anomalies (Class $\mathrm{H}_{V}$ ) (see [12] for more details), and are totally determined up to some constants.

(ii) Nonhydrostatic transport (Classes D and D): These are solutions of Eq. (9) made purely out of nonhydrostatic tensor structures. They are best expressed by introducing a symmetric covariant derivative operator $\mathrm{D}_{M_{1} M_{2} \ldots M_{n}}^{n}=\nabla_{\left(M_{1}\right.} \nabla_{M_{2}} \ldots \nabla_{\left.M_{n}\right)}$. They form a basis for arbitrary derivative operators because antisymmetric combinations can always be replaced by combinations of Riemann curvature tensor. We can now write down the most generic nonhydrostatic constitutive relations as combinations of $\mathrm{D}^{n} \delta_{\mathcal{B}} g_{R S}$ for all $n$. A particularly convenient parametrization is

$$
\begin{aligned}
T_{\text {non-hydrostatic }}^{M N}= & -\frac{1}{2} \sum_{n=0}^{\infty}\left[\mathfrak{夭}_{n}^{(M N)(R S)} \frac{1}{2} \mathrm{D}^{n} \delta_{\mathcal{B}} g_{R S}\right. \\
& \left.+\mathrm{D}^{n}\left(\mathfrak{\mho}_{n}^{(M N)(R S)} \frac{1}{2} \delta_{\mathcal{B}} g_{R S}\right)\right],
\end{aligned}
$$

where $\mathfrak{C}_{n}^{(M N)(R S)}$ is an arbitrary matrix with $n$ additional symmetric indices to be contracted with $\mathrm{D}^{n}$. Note that when $\mathrm{D}^{n}$ in the second term hits $\delta_{\mathcal{B}} g_{R S}$, we get the same term as the first one. All the other terms coming out of differentiation by parts have merely been included for convenience, as we shall see. Some minor comments about the structure of $\mathfrak{C}_{n}^{(M N)(R S)}:$ (1) It cannot contain an instance of $\mathrm{D}^{m} g_{A B}$ for $m>n$ as the respective terms in Eq. (14) are taken care of in the $\mathfrak{S}_{m}^{(M N)(R S)}$ term. (2) For an instance of $\mathrm{D}^{n} g_{A B}$ in $\mathfrak{夭}_{n}^{(M N)(R S)}$, we must be careful not to overcount the terms gained by $(M N) \leftrightarrow(A B)$ exchange, which would give the same contribution to $T_{\text {non-hydrostatic }}^{M N}$ in Eq. (14).

We can make our lives much easier by thinking of $\mathfrak{C}_{n}^{(M N)(R S)}$ as a $\frac{1}{2}(d+1)(d+2)$ dimensional matrix, and $T_{\mathrm{D} \cup \overline{\mathrm{D}}}^{M N}$ and $\delta_{\mathcal{B}} g_{R S}$ as column vectors. By suppressing the indices in this notation, the above expression becomes

$T_{\text {nonhydrostatic }}=-\frac{1}{4} \sum_{n=0}^{\infty}\left[\mathfrak{\mho}_{n} \cdot \mathrm{D}^{n} \delta_{\mathcal{B}} g+\mathrm{D}^{n}\left(\mathfrak{C}_{n} \cdot \delta_{\mathcal{B}} g\right)\right]$.

Using differentiation by parts on the second term we can compute 


$$
\begin{aligned}
\frac{1}{2} \delta_{\mathcal{B}} g^{\mathrm{T}} \cdot T_{\text {nonhydrostatic }} & =-\frac{1}{8} \sum_{n=0}^{\infty}\left[\delta_{\mathcal{B}} g^{\mathrm{T}} \cdot \mathfrak{C}_{n} \cdot \mathrm{D}^{n} \delta_{\mathcal{B}} g+\delta_{\mathcal{B}} g^{\mathrm{T}} \cdot \mathrm{D}^{n}\left(\mathfrak{C}_{n} \cdot \delta_{\mathcal{B}} g\right)\right] \\
& =-\frac{1}{8} \delta_{\mathcal{B}} g^{\mathrm{T}} \cdot \sum_{n=0}^{\infty}\left(\mathfrak{C}_{n}^{\mathrm{T}}+(-)^{n} \mathfrak{C}_{n}^{\mathrm{T}}\right) \cdot \mathrm{D}^{n} \delta_{\mathcal{B}} g+\nabla_{M} \Theta_{\mathfrak{C}}^{M}, \\
& =-\frac{1}{4} \delta_{\mathcal{B}} g^{\mathrm{T}} \cdot \sum_{n=0}^{\infty} \mathfrak{D}_{n} \cdot \mathrm{D}^{n} \delta_{\mathcal{B}} g+\nabla_{M} \Theta_{\mathfrak{D}}^{M}+\nabla_{M} \Theta_{\mathfrak{D}}^{M} .
\end{aligned}
$$

In the last line we have split $\mathfrak{夭}_{n}$ into

$$
\mathfrak{D}_{n}=\frac{1}{2}\left(\mathfrak{C}_{n}+(-)^{n} \mathfrak{C}_{n}^{\mathrm{T}}\right), \quad \overline{\mathfrak{D}}_{n}=\frac{1}{2}\left(\mathfrak{C}_{n}-(-)^{n} \mathfrak{C}_{n}^{\mathrm{T}}\right),
$$

and the corresponding contribution to $\Theta_{\mathfrak{C}}^{M}$ into $\Theta_{\mathfrak{D}}^{M}$ and $\Theta_{\bar{D}}^{M}$. Note that for the constitutive relations coupled to $\overline{\mathfrak{D}}_{n}$, called Class $\overline{\mathrm{D}}$ for non-dissipative, the story pretty much ends here. We can infer from Eq. (16) that the associated constitutive relations $T_{\overline{\mathrm{D}}}^{M N}$ satisfy Eq. (9) with $N^{M}=\Theta_{\overline{\mathfrak{D}}}^{M}$ and $\Delta=0$.
They correspond to nonhydrostatic transport that does not cause any dissipation.

For the constitutive relations coupled to $\mathfrak{D}_{n}$ however, called Class D for dissipative, we need to do little more work to ensure that the associated $\Delta$ can be made positive definite. To do that, we rewrite the $\mathfrak{D}_{n}$ part of Eq. (16) as

$$
\begin{aligned}
\frac{1}{2} \delta_{\mathcal{B}} g^{\mathrm{T}} \cdot T_{\mathrm{D}} & =-\frac{1}{4} \delta_{\mathcal{B}} g^{\mathrm{T}} \cdot \mathfrak{D}_{0(0)} \cdot \delta_{\mathcal{B}} g-\frac{1}{2} \delta_{\mathcal{B}} g^{\mathrm{T}} \cdot \mathfrak{D}_{0(0)} \cdot\left(\Upsilon_{1} \cdot \delta_{\mathcal{B}} g\right)+\nabla_{M} \Theta_{\mathfrak{D}}^{M} \\
& =-\underbrace{\frac{1}{4}\left(\left(1+\Upsilon_{1}\right) \cdot \delta_{\mathcal{B}} g\right)^{\mathrm{T}} \cdot \mathfrak{D}_{0(0)} \cdot\left(\left(1+\Upsilon_{1}\right) \cdot \delta_{\mathcal{B}} g\right)}_{\text {quadratic form }}+\underbrace{\frac{1}{4}\left(\Upsilon_{1} \cdot \delta_{\mathcal{B}} g\right)^{\mathrm{T}} \cdot \mathfrak{D}_{0(0)} \cdot\left(\Upsilon_{1} \cdot \delta_{\mathcal{B}} g\right)}_{\text {residue }}+\underbrace{\nabla_{M} \Theta_{\mathfrak{D}}^{M}}_{\text {total derivative }},
\end{aligned}
$$

where $\mathfrak{D}_{0(n)}$ denotes the $n$th derivative piece in $\mathfrak{D}_{(0)}$, while $\Upsilon_{1}$ is a differential operator

$$
\Upsilon_{1}=\frac{1}{2} \mathfrak{D}_{0(0)}^{-1} \sum_{n=1}^{\infty}\left(\mathfrak{D}_{0(n)}+\mathfrak{D}_{n} \mathrm{D}^{n}\right)
$$

The quadratic form piece in Eq. (18) is of most interest to us, as it contributes to $\Delta$. The total derivative piece on the other hand is a contribution to the free energy current $N^{M}$. Finally, the residue piece is what we will like to get rid of. Note that every term in $\Upsilon_{1}$ has at least one derivative. Consequently, the residue piece is at least 4 order in derivatives. If we are only interested in the constitutive relations up to second derivative order, we can ignore this piece altogether. However, we will illustrate the full procedure here for completeness. Using differentiation by parts, the residue piece can be rewritten as

$$
\frac{1}{4}\left(\Upsilon_{1} \cdot \delta_{\mathcal{B}} g\right)^{\mathrm{T}} \cdot \mathfrak{D}_{0(0)} \cdot\left(\Upsilon_{1} \cdot \delta_{\mathcal{B}} g\right)=\frac{1}{4} \delta_{\mathcal{B}} g^{\mathrm{T}} \cdot\left(\Upsilon_{1}^{\dagger} \cdot \mathfrak{D}_{0(0)} \cdot \Upsilon_{1} \cdot \delta_{\mathcal{B}} g\right)+\nabla_{M} \Theta_{\mathfrak{D}, 1}^{M} \cdot
$$

Putting this back in Eq. (18) we get

$$
\begin{aligned}
\frac{1}{2} \delta_{\mathcal{B}} g^{\mathrm{T}} \cdot T_{\mathrm{D}}= & -\underbrace{\frac{1}{4}\left(\left(1+\Upsilon_{1}+\Upsilon_{2}\right) \cdot \delta_{\mathcal{B}} g\right)^{\mathrm{T}} \cdot \mathfrak{D}_{0(0)} \cdot\left(\left(1+\Upsilon_{1}+\Upsilon_{2}\right) \cdot \delta_{\mathcal{B}} g\right)}_{\text {quadratic form }} \\
& +\underbrace{\frac{1}{2}\left(\left(\Upsilon_{1}+\frac{1}{2} \Upsilon_{2}\right) \cdot \delta_{\mathcal{B}} g\right)^{\mathrm{T}} \cdot \mathfrak{D}_{0(0)} \cdot\left(\Upsilon_{2} \cdot \delta_{\mathcal{B}} g\right)}_{\text {residue }}+\underbrace{\nabla_{M}\left(\Theta_{\mathfrak{D}}^{M}+\Theta_{\mathfrak{D}, 1}^{M}\right)}_{\text {total derivative }},
\end{aligned}
$$


where $\Upsilon_{2}$ is another derivative operator

$$
\Upsilon_{2}=-\frac{1}{2} \mathfrak{D}_{0(0)}^{-1} \cdot \Upsilon_{1}^{\dagger} \cdot \mathfrak{D}_{0(0)} \cdot \Upsilon_{1}
$$

Comparing Eqs. (21) to (18), hopefully the reader can see a pattern. The quadratic form piece now has some additional higher derivative terms, whereas we have pushed the residue piece to 5 th derivative order. We can repeat this procedure iteratively to push the residue piece to arbitrarily high derivative orders and obtain

$$
\frac{1}{2} \delta_{\mathcal{B}} g^{\mathrm{T}} \cdot T_{\mathrm{D}}=-\underbrace{-\frac{1}{4}\left(\left(1+\sum_{n=1}^{\infty} \Upsilon_{n}\right) \cdot \delta_{\mathcal{B}} g\right)^{\mathrm{T}} \cdot \mathfrak{D}_{0(0)} \cdot\left(\left(1+\sum_{n=1}^{\infty} \Upsilon_{n}\right) \cdot \delta_{\mathcal{B}} g\right)}_{\text {quadratic form }}+\underbrace{\nabla_{M}\left(\Theta_{\mathfrak{D}}^{M}+\sum_{n=1}^{\infty} \Theta_{\mathfrak{D}, n}^{M}\right)}_{\text {total derivative }},
$$

where

$$
\left.\Upsilon_{d+1}\right|_{d=1} ^{\infty}=-\mathfrak{D}_{0(0)}^{-1} \cdot\left(\sum_{k=1}^{d-1} \Upsilon_{k}^{\dagger}+\frac{1}{2} \Upsilon_{d}^{\dagger}\right)\left(\mathfrak{D}_{0(0)} \cdot \Upsilon_{d}\right)
$$

We see therefore that the Class D constitutive relations satisfy Eq. (9) with

$$
\Delta=\frac{1}{4}\left(\left(1+\sum_{n=1}^{\infty} \Upsilon_{n}\right) \cdot \delta_{\mathcal{B}} g\right)^{\mathrm{T}} \cdot \mathfrak{D}_{0(0)} \cdot\left(\left(1+\sum_{n=1}^{\infty} \Upsilon_{n}\right) \cdot \delta_{\mathcal{B}} g\right)
$$

and the free energy current

$$
N^{M}=\Theta_{\mathfrak{D}}^{M}+\sum_{n=1}^{\infty} \Theta_{\mathfrak{D}, n}^{M}
$$

The condition $\Delta \geq 0$ therefore, only gives a constraint on the first derivative constitutive relations by forcing all the eigenvalues of $\mathfrak{D}_{0(0)}$ to be nonnegative. See [22] for more details on this. We do not get any further constraints from the second law at higher derivative orders in the nonhydrostatic sector.

Recall that to avoid the overcounting of constitutive relations related to each other by combinations of equations of motion, we had decided to drop all the constitutive relations that involve $u^{M} \delta_{\mathcal{B}} g_{M N}$. To respect this, we must demand that none of the $(M N)(R S)$ indices in $\mathfrak{C}^{(M N)(R S)}$ should come from a $u^{M}$. Equivalently $\mathfrak{C}^{(M N)(R S)} V_{M}=\mathfrak{c}^{(M N)(R S)} V_{R}=0$. Furthermore, $\mathfrak{c}^{(M N)(R S)}$ should of course not have an explicit occurrence of $u^{M} \delta_{\mathcal{B}} g_{M N}$.

\section{Results up to first order}

In $[11,13]$, we discussed null fluids up to first order in derivatives. We briefly recollect these results here and illustrate how they fit into the classification presented in the previous subsection. To setup the notation, we enlist the independent fluid data at first order in Table I. (i) Hydrostatic transport (Class $\mathrm{H}_{\mathrm{S}}$ ): The most generic hydrostatic scalar up to first derivative order is simply

$$
\mathcal{N}=P\left(T, \mu_{m}\right)
$$

where $P\left(T, \mu_{m}\right)$ is identified with the pressure of the fluid. Interestingly, there are no hydrostatic

TABLE I. First order fluid data. Note that we have defined two

\begin{tabular}{|c|c|c|}
\hline \multicolumn{3}{|c|}{ Nonhydrostatic_-onshell independent } \\
\hline$\Theta$ & $\frac{T}{2} P^{M N} \delta_{\mathcal{B}} g_{M N}$ & $\nabla_{M} u^{M}$ \\
\hline$\tau^{M}, \bar{\tau}^{M}$ & $T P^{M N} V^{R} \delta_{\mathcal{B}} g_{\mathrm{NR}}$ & $P^{M N} \frac{1}{T} \partial_{N} T$ \\
\hline$\sigma^{M N}$ & $T P^{R\langle M} P^{N\rangle S} \delta_{\mathcal{B}} g_{R S}$ & $2 P^{R\langle M} P^{N\rangle S} \nabla_{R} u_{S}$ \\
\hline \multicolumn{3}{|c|}{ Nonhydrostatic_-onshell dependent } \\
\hline$\Theta_{T}$ & $T u^{M} V^{N} \delta_{\mathcal{B}} g_{M N}$ & $u^{M} \frac{1}{T} \partial_{M} T$ \\
\hline$\Theta_{\varpi}$ & $\frac{T}{2} u^{M} u^{N} \delta_{\mathcal{B}} g_{M N}$ & $u^{M} T \partial_{M} \varpi$ \\
\hline$\tau_{\varpi}^{M}$ & $T P^{M N} u^{R} \delta_{\mathcal{B}} g_{N R}$ & $P^{M N}\left(T \partial_{N} \varpi+u^{R} \nabla_{R} u_{N}\right)$ \\
\hline \multicolumn{3}{|c|}{ Hydrostatic } \\
\hline $\bar{\tau}_{\varpi}^{M}$ & \multicolumn{2}{|c|}{$P^{M N} T \partial_{N} \varpi$} \\
\hline$\omega^{M N}$ & \multicolumn{2}{|c|}{$2 P^{R[M} P^{N] S} \nabla_{R} u_{S}$} \\
\hline
\end{tabular}
symbols $\tau^{M}$ and $\bar{\tau}^{M}$ for the term $P^{M N} \frac{1}{T} \partial_{N} T$. This is to acknowledge the fact that in the presence of torsion, the term $\bar{\tau}^{M}=P^{M N} \frac{1}{T} \partial_{N} T$ is actually hydrostatic, while $\tau^{M}=P^{M N}\left(\frac{1}{T} \partial_{N} T+u^{R} H_{R N}\right)$ is nonhydrostatic. This distinction will be handy later. We will also use the acceleration $a^{M}=u^{N} \nabla_{N} u^{M}=\tau_{\varpi}^{M}-\bar{\tau}_{\varpi}^{M}$ sometimes. 
scalars involving just one derivative. We can use Eq. (13) and find the associated constitutive relations, which basically gives the entire ideal null fluid

$T_{\mathrm{H}_{S}}^{M N}=R u^{M} u^{N}+2 E u^{(M} V^{N)}+P P^{M N}+\mathcal{O}\left(\partial^{2}\right)$,

with $\Theta_{N}^{M}=0$. Here $P^{M N}=g^{M N}+2 u^{(M} V^{N)}$ is a projector transverse to $u^{M}$ and $V^{M} . R=\frac{1}{T} \frac{\partial P}{\partial \varpi}$ is identified with the mass density and $E=T \frac{\partial P}{\partial T}-P$ with the energy density of the fluid. Together they define the thermodynamic relations

$\mathrm{d} E=T \mathrm{~d} S+\mu_{m} \mathrm{~d} R, \quad E+P=S T+R \mu_{m}$,

where $S$ is called the entropy density.

(ii) Nonhydrostatic transport (Classes D and D̄): Looking back at Eq. (14), we can infer that since $\delta_{\mathcal{B}} g_{M N}$ already contains a derivative, at first derivative order we only need a zero-derivative tensor $\mathfrak{G}_{0}^{(M N)(R S)}$. It takes the most generic form

$$
\begin{aligned}
\mathfrak{夭}_{0}^{(M N)(R S)}= & 2 T \eta P^{M\langle R} P^{S\rangle N}+T \zeta P^{M N} P^{R S} \\
& +4 T^{2} \kappa V^{(M} P^{N)(R} V^{S)} .
\end{aligned}
$$

The first thing to note here is that all the terms in Eq. (30) are symmetric under the exchange of $(M N) \leftrightarrow(R S)$. Consequently, the zero derivative $\overline{\mathfrak{D}}_{0}$ and hence the first derivative Class $\overline{\mathrm{D}}$ constitutive relations are identically zero,

$$
T_{\overline{\mathrm{D}}}^{M N}=\mathcal{O}\left(\partial^{2}\right), \quad N_{\overline{\mathrm{D}}}^{M}=\mathcal{O}\left(\partial^{2}\right) .
$$

On the other hand, for Class D we get

$$
T_{\mathrm{D}}^{M N}=-\eta \sigma^{M N}-\zeta P^{M N} \Theta-2 T \kappa_{\epsilon} V^{(M} \tau^{N)}+\mathcal{O}\left(\partial^{2}\right),
$$

with

$$
\begin{aligned}
N_{\mathrm{D}}^{M} & =\mathcal{O}\left(\partial^{2}\right), \\
\Delta & =\frac{1}{2 T} \eta \sigma^{M N} \sigma_{M N}+\frac{1}{T} \zeta \Theta^{2}+\kappa_{\epsilon} \tau^{M} \tau_{M} .
\end{aligned}
$$

The condition $\Delta \geq 0$ simply implies that all the transport coefficients are non-negative

$$
\eta, \quad \zeta, \quad \kappa \geq 0 .
$$

We can identify these transport coefficients as: $\eta$ shear viscosity, $\zeta$ bulk viscosity, and $\kappa$ thermal conductivity of the fluid.
The full set of constitutive relations up to first derivative order are a direct sum of Eqs. (28), (31), and (32), giving us

$$
\begin{aligned}
T^{M N}= & R u^{M} u^{N}+2 E u^{(M} V^{N)}+P P^{M N}-\eta \sigma^{M N} \\
& -\zeta P^{M N} \Theta-2 T \kappa_{\epsilon} V^{(M} \tau^{N)}+\mathcal{O}\left(\partial^{2}\right) .
\end{aligned}
$$

They are supported by the free energy and entropy currents

$$
\begin{aligned}
N^{M} & =\frac{1}{T} P u^{M}+\mathcal{O}\left(\partial^{2}\right), \\
J_{S}^{M} & =N^{M}-T^{M N} \beta_{N}=S u^{M}-\kappa_{\epsilon} \tau^{M}+\mathcal{O}\left(\partial^{2}\right) .
\end{aligned}
$$

The transport coefficients follow the thermodynamic constraints in Eq. (29) at ideal order and the positivity relations Eq. (34) at first derivative order.

This finishes our crash course in null fluids. We still need to discuss the translation of these results to Galilean fluids, which we will come back to in Sec. IV. In the next section, we will use the machinery developed here to write down the null fluid constitutive relations up to second derivative order.

\section{SECOND ORDER NULL FLUIDS}

In the previous section we gave a self-contained review of null fluids and presented an algorithm to generate the respective constitutive relations up to arbitrarily high derivative orders. The goal of this section is to use this algorithm to write down the null fluid constitutive relations up to second order. Apart from $P, \eta, \zeta$, and $\kappa$ at previous orders, we find a total of 25 transport coefficients: 5 in Class $\mathrm{H}_{S}, 9$ in Class D, and 11 in Class $\overline{\mathrm{D}}$. For readers who are only interested in the results, they have been summarized in Tables II-VI. In the remaining of this section, we will explain how we arrived at these results.

TABLE II. Class $\mathrm{H}_{S}$ constitutive relations up to second derivative order. We have defined a differential operator $\mathfrak{g}^{M N}=$ $g^{M N}+2 T u^{(M} V^{N)} \frac{\partial}{\partial T}+\frac{1}{T} u^{M} u^{N} \frac{\partial}{\partial \varpi}$ for brevity.

\begin{tabular}{lc}
\hline \hline Coefficient & $T^{M N} \ni \frac{2}{\sqrt{-g}} \frac{\delta(\sqrt{-g} \mathcal{N})}{\delta g_{M N}}=\mathcal{N} g^{M N}+2 \frac{\delta \mathcal{N}}{\delta g_{M N}}$ \\
\hline$P$ & $\mathfrak{g}^{M N} P \equiv R u^{M} u^{N}+2 E u^{(M} V^{N)}+P P^{M N}$ \\
$\alpha_{1}$ & $\frac{1}{2 T^{2}} \bar{\tau}_{\varpi}^{R} \bar{\tau}_{\varpi R} \mathfrak{g}^{M N}\left(\alpha_{1} T^{2}\right)-\frac{1}{T} \nabla_{R}\left(\alpha_{1} T \bar{\tau}_{\varpi}^{R}\right) u^{M} u^{N}$ \\
& $+2 \alpha_{1} V^{(M} \bar{\tau}_{\varpi}^{N)} \Theta_{\varpi}-\alpha_{1} \bar{\tau}_{\varpi}^{(M} \bar{\tau}_{\varpi}^{N)}$ \\
$\alpha_{2}$ & $\bar{\tau}_{\varpi}^{R} \bar{\tau}_{R} \mathfrak{g}^{M N} \alpha_{2}-2 T \nabla_{R}\left(\frac{\alpha_{2}}{T} \bar{\tau}_{\varpi}^{R}\right) u^{(M} V^{N)}-\frac{1}{T} \nabla_{R}\left(\alpha_{2} T \bar{\tau}^{R}\right) u^{M} u^{N}$ \\
& $+2 \alpha_{2} V^{(M} \bar{\tau}_{\varpi}^{N)} \Theta_{T}+2 \alpha_{2} V^{(M} \bar{\tau}^{N)} \Theta_{\varpi}-2 \alpha_{2} \bar{\tau}^{(M} \bar{\tau}_{\varpi}^{N)}$ \\
$\alpha_{3}$ & $\frac{1}{4} \omega^{R S} \omega_{R S} \mathfrak{g}^{M N} \alpha_{3}-\alpha_{3} \omega^{M}{ }_{R} \omega^{N R}+2 \alpha_{3} V^{(M} \omega^{N) R} a_{R}$ \\
& $-2 \nabla_{R}\left(\alpha_{3} \omega^{R S}\right) P_{S}{ }^{(M} u^{N)}$ \\
$\alpha_{4}$ & $-2 \nabla_{R}\left(\alpha_{4} \omega^{R S}\right) P_{S}\left(M V^{N)}\right.$ \\
$\alpha_{5}$ & $R \mathfrak{g}^{M N} \alpha_{5}-2 R^{M N} \alpha_{5}+2 \nabla^{M} \nabla^{N} \alpha_{5}-2 g^{M N} \nabla^{R} \nabla_{R} \alpha_{5}$ \\
\hline \hline
\end{tabular}


TABLE III. Class $\mathrm{H}_{S}$ free energy current up to second derivative order.

\begin{tabular}{lc}
\hline \hline Coefficient & $N^{M} \ni \mathcal{N} \beta^{M}+\Theta_{\mathcal{N}}^{M}$ \\
\hline$P$ & $\frac{1}{T} P u^{M}$ \\
$\alpha_{1}$ & $\frac{\alpha_{1}}{T}\left(\frac{1}{2} u^{M} \bar{\tau}_{\varpi}^{R} \bar{\tau}_{\varpi R}-\bar{\tau}_{\varpi}^{M} \Theta_{\varpi}\right)$ \\
$\alpha_{2}$ & $\frac{\alpha_{2}}{T}\left(u^{M} \bar{\tau}_{\varpi}^{R} \bar{\tau}_{R}-\bar{\tau}^{M} \Theta_{\varpi}-\bar{\tau}_{\varpi}^{M} \Theta_{T}\right)$ \\
$\alpha_{3}$ & $\frac{\alpha_{3}}{T}\left[\frac{1}{4} u^{M} \omega^{R S} \omega_{R S}-\omega^{M N} \tau_{\varpi N}\right]$ \\
$\alpha_{4}$ & $-\frac{\alpha_{4}}{T} \omega^{M N} \tau_{N}$ \\
$\alpha_{5}$ & $\frac{\alpha_{5}}{T} R u^{M}-2 \alpha_{5} \nabla_{R} \nabla^{(R}\left(\frac{\left.u^{M}\right)}{T}\right)+2 \nabla^{(R}\left(\frac{u^{M)}}{T}\right) \partial_{R} \alpha_{5}$ \\
& $+2 \alpha_{5} \nabla^{M} \nabla_{R}\left(\frac{u^{R}}{T}\right)-2 \nabla_{R}\left(\frac{u^{R}}{T}\right) \nabla^{M} \alpha_{5}$ \\
\hline \hline
\end{tabular}

\section{A. Hydrostatic transport (Class $\mathbf{H}_{\mathbf{S}}$ )}

Let us start with Class $\mathrm{H}_{S}$. As discussed in Sec. II C, this class is characterized by the most generic hydrostatic scalar $\mathcal{N}$ up to some total derivative terms. Up to second derivative order, we have 5 such terms apart from the ideal order pressure

$$
\begin{aligned}
\mathcal{N}= & P+\frac{1}{2} \alpha_{1} \bar{\tau}_{\varpi}^{M} \bar{\tau}_{\varpi M}+\alpha_{2} \bar{\tau}_{\varpi}^{M} \tau_{M}+\frac{1}{4} \alpha_{3} \omega^{M N} \omega_{M N} \\
& +\frac{1}{2} \alpha_{4} \omega^{M N} H_{M N}+\alpha_{5} R+\mathcal{O}\left(\partial^{3}\right),
\end{aligned}
$$

where $R$ is the Ricci scalar. Note the second and fourth terms in particular: the former is nonhydrostatic while the latter vanishes. However, their variations do contribute to hydrostatic constitutive relations as we shall see in the following. This is an instance of the "unnaturalness" arising due to the absence of torsion we talked about around Eq. (4). In the presence of torsion, both of these terms are actually hydrostatic. The astute reader might note that by this token we should also include the quadratic terms coupling to $\tau^{M} \tau_{M}$ and $H^{M N} H_{M N}$. However, in the absence of torsion their variations only lead to nonhydrostatic constitutive relations and thus can be ignored here.

Varying $\mathcal{N}$ in Eq. (37) and using the formulas in Eq. (13), we can now read out the Class $\mathrm{H}_{S}$ constitutive relations and free energy current. Note that the $\delta_{\mathcal{B}}$ variation of fluid variables can be represented in terms of $\delta_{\mathcal{B}} g_{M N}$ as

$$
\begin{aligned}
\delta_{\mathcal{B}} T & =T V^{M} u^{N} \delta_{\mathcal{B}} g_{M N}, \quad \delta_{\mathcal{B}} \varpi=\frac{1}{2 T} u^{M} u^{N} \delta_{\mathcal{B}} g_{M N}, \\
\delta_{\mathcal{B}} u^{M} & =\left(2 u^{M} V^{(R} u^{S)}+V^{M} u^{R} u^{S}\right) \frac{1}{2} \delta_{\mathcal{B}} g_{R S}, \\
\delta_{\mathcal{B}} u_{M} & =\left(-V_{M} u^{R} u^{S}+2 P_{M}{ }^{(R} u^{S)}\right) \frac{1}{2} \delta_{\mathcal{B}} g_{R S}, \\
\delta_{\mathcal{B}} V_{M} & =\left(-2 V_{M} u^{(R} V^{S)}+2 P_{M}{ }^{(R} V^{S)}\right) \frac{1}{2} \delta_{\mathcal{B}} g_{R S},
\end{aligned}
$$

while that of the Ricci scalar as

$\delta_{\mathcal{B}} R=\left(-2 R^{M N}+2 \nabla^{(M} \nabla^{N)}-2 g^{M N} \nabla^{R} \nabla_{R}\right) \frac{1}{2} \delta_{\mathcal{B}} g_{R S}$.

These will be useful in the variational calculation. We have

\begin{tabular}{|c|c|c|c|}
\hline Coefficient & \multicolumn{2}{|c|}{$\mathfrak{G}_{0}^{(M N)(R S)}$} & $T^{M N} \ni-\mathfrak{C}_{0}^{(M N)(R S)} \frac{1}{2} \delta_{\mathcal{B}} g_{R S}$ \\
\hline$\eta$ & \multicolumn{2}{|c|}{$2 T \eta P^{M\langle R} P^{S\rangle N}$} & $-\eta \sigma^{M N}$ \\
\hline$\zeta$ & \multicolumn{2}{|c|}{$T \zeta P^{M N} P^{R S}$} & $-\zeta P^{M N} \Theta$ \\
\hline$\kappa T$ & \multicolumn{2}{|c|}{$4 T^{2} \kappa V^{(M} P^{N)(R} V^{S)}$} & $-2 T \kappa V^{(M} \tau^{N)}$ \\
\hline$\delta_{1}^{+}$ & $2 T^{2} \delta_{1}^{+}\left(V^{(M} P^{N)\langle R} P^{S\rangle(A} V^{B)}\right) \delta_{\mathcal{B}} g_{A B}$ & $2 T \delta_{1}^{+} V^{(M} P^{N)\langle R} \tau^{S\rangle}$ & $-\delta_{1}^{+} V^{(M} \sigma^{N) R} \tau_{R}$ \\
\hline$\delta_{1}^{-}$ & $2 T^{2} \delta_{1}^{-}\left(V^{(R} P^{S)\langle M} P^{N\rangle\left({ }^{A}\right.} V^{B)}\right) \delta_{\mathcal{B}} g_{A B}$ & $2 T \delta_{1}^{-} \tau^{\langle M} P^{N\rangle(R} V^{S)}$ & $-\delta_{1}^{-} \tau^{\langle M} \tau^{N\rangle}$ \\
\hline$\delta_{2}^{+}$ & $2 T^{2} \delta_{2}^{+}\left(V^{(M} P^{N)(A} V^{B)} P^{R S}\right) \delta_{\mathcal{B}} g_{A B}$ & $2 T \delta_{2}^{+} V^{(M} \tau^{N)} P^{R S}$ & $-2 \delta_{2}^{+} V^{(M} \tau^{N)} \Theta$ \\
\hline$\delta_{2}^{-}$ & $2 T^{2} \delta_{2}^{-}\left(V^{(R} P^{S)(A} V^{B)} P^{M N}\right) \delta_{\mathcal{B}} g_{A B}$ & $2 T \delta_{2}^{-} P^{M N} V^{(R} \tau^{S)}$ & $-\delta_{2}^{-} P^{M N} \tau^{R} \tau_{R}$ \\
\hline$\delta_{3}^{+}$ & $2 T^{2} \delta_{3}^{+}\left(P^{M\langle A} P^{B\rangle N} P^{R S}\right) \delta_{\mathcal{B}} g_{A B}$ & $2 T \delta_{3}^{+} \sigma^{M N} P^{R S}$ & $-2 \delta_{3}^{+} \sigma^{M N} \Theta$ \\
\hline$\delta_{3}^{-}$ & $2 T^{2} \delta_{3}^{-}\left(P^{R\langle A} P^{B\rangle S} P^{M N}\right) \delta_{\mathcal{B}} g_{A B}$ & $2 T \delta_{3}^{-} P^{M N} \sigma^{R S}$ & $-\delta_{3}^{-} P^{M N} \sigma^{R S} \sigma_{R S}$ \\
\hline$\delta_{4}$ & $2 T^{2} \delta_{4}\left(P^{A\langle M} P^{B\langle R} P^{S\rangle N\rangle}\right) \delta_{\mathcal{B}} g_{A B}$ & $2 T \delta_{4} \sigma^{\langle M\langle R} P^{S\rangle N\rangle}$ & $\left.-\delta_{4} \sigma^{\langle M R} \sigma_{R} N\right\rangle$ \\
\hline$\delta_{5}$ & $T^{2} \delta_{5}\left(P^{M N} P^{R S} P^{A B}\right) \delta_{\mathcal{B}} g_{A B}$ & $2 T \delta_{5} P^{M N} P^{R S} \Theta$ & $-2 \delta_{5} P^{M N} \Theta^{2}$ \\
\hline$\delta_{6}^{+}$ & \multicolumn{2}{|c|}{$2 T \delta_{6}^{+} V^{(M} P^{N)\langle R} \bar{\tau}_{\varpi}^{S\rangle}$} & $-\delta_{6}^{+} V^{(M} \sigma^{N) R} \bar{\tau}_{\varpi R}$ \\
\hline$\delta_{6}^{-}$ & \multicolumn{2}{|c|}{$2 T^{2} \delta_{6}^{-} \bar{\tau}_{\varpi}^{\langle M} P^{N\rangle(R} V^{S)}$} & $-\delta_{6}^{-} \bar{\tau}_{\varpi}^{\langle M} \tau^{N\rangle}$ \\
\hline$\delta_{7}^{+}$ & \multicolumn{2}{|c|}{$2 T^{2} \delta_{7}^{+} V^{(M} \bar{\tau}_{\varpi}^{N)} P^{R S}$} & $-2 \delta_{7}^{+} V^{(M} \bar{\tau}_{\varpi}^{N)} \Theta$ \\
\hline$\delta_{7}^{-}$ & \multicolumn{2}{|c|}{$2 T^{2} \delta_{7}^{-} P^{M N} V^{(R} \bar{\tau}_{\varpi}^{S)}$} & $-\delta_{7}^{-} P^{M N} \bar{\tau}_{\varpi}^{R} \tau_{R}$ \\
\hline $\bar{\delta}_{8}$ & \multicolumn{2}{|c|}{$2 T \bar{\delta}_{8} V^{(M} \omega^{N)(R} V^{S)}$} & $-\bar{\delta}_{8} V^{(M} \omega^{N) R} \tau_{R}$ \\
\hline $\bar{\delta}_{9}$ & \multicolumn{2}{|c|}{$2 T \bar{\delta}_{9} \omega^{\langle M\langle R} P^{S\rangle N\rangle}$} & $-\bar{\delta}_{9} \omega^{\langle M R} \sigma_{R}{ }^{N\rangle}$ \\
\hline
\end{tabular}
summarized our results in Tables II and III. They enlist the term by term contribution to $T^{M N}$ and $N^{M}$ coming from

TABLE IV. Terms in $\mathfrak{E}_{0}^{(M N)(R S)}$ with at most one derivative and their contribution to the second order Class D and $\overline{\mathrm{D}}$ constitutive relations. Here we have defined $\delta_{i}^{ \pm}=\left(\delta_{i} \pm \bar{\delta}_{i}\right) / 2$. The transport coefficients $\delta_{i}$ couple to Class D constitutive relations while $\bar{\delta}_{i}$ couples to Class $\overline{\mathrm{D}}$. 
varying Eq. (37). The full Class $\mathrm{H}_{S}$ constitutive relations and free energy current will be a direct sum of all these contributions.

\section{B. Nonhydrostatic transport (Classes D and $\overline{\mathbf{D}})$}

Up to second derivative order, the nonhydrostatic constitutive relations in Eq. (14) get contributions from a zero derivative tensor $\mathfrak{C}_{1}^{(M N)(R S) T}$ and a tensor $\mathfrak{C}_{0}^{(M N)(R S)}$ with at most one derivative

$$
\begin{aligned}
T_{\mathrm{D} \cup \overline{\mathrm{D}}}^{M N}= & -\mathfrak{C}_{0}^{(M N)(R S)} \frac{1}{2} \delta_{\mathcal{B}} g_{R S}-\mathfrak{G}_{1}^{(M N)(R S) T} \frac{1}{2} \nabla_{T} \delta_{\mathcal{B}} g_{R S} \\
& -\frac{1}{2} \nabla_{T} \mathfrak{夭}_{1}^{(M N)(R S) T} \frac{1}{2} \delta_{\mathcal{B}} g_{R S}+\mathcal{O}\left(\partial^{3}\right) .
\end{aligned}
$$

The respective contribution to the free energy current truncated to two derivatives is given as

$$
N_{\mathrm{DU} \overline{\mathrm{D}}}^{M}=-\frac{1}{8} \boldsymbol{\mho}_{1}^{(R S)(A B) M} \delta_{\mathcal{B}} g_{R S} \delta_{\mathcal{B}} g_{A B}+\mathcal{O}\left(\partial^{3}\right) .
$$

Interestingly, it is only nonvanishing for the Class $\overline{\mathrm{D}}$ constitutive relations associated with $\overline{\mathfrak{D}}_{1}^{(R S)(A B) M}=$ $\mathfrak{夭}_{1}^{(R S)(A B) M}$. Class D constitutive relations up to second derivative order do not need any free-energy transport.

Let us start our discussion with $\mathfrak{\mathfrak { V }}_{0}$. We need the most generic 4-tensor $\mathfrak{E}_{0}^{(M N)(R S)}$ made out of 0 and 1-derivative data with appropriate symmetry properties. Note that none of the indices can come from a $u^{M}$ because the respective terms are eliminated by the equations of motion. Note also that there are 1-derivative terms in $\mathfrak{夭}_{0}$ which are constructed using $\delta_{\mathcal{B}} g_{M N}$, i.e., $\mathfrak{G}_{0}^{(M N)(R S)(A B)} \delta_{\mathcal{B}} g_{A B} \in \mathfrak{C}_{0}^{(M N)(R S)}$. The respective contribution to $T^{M N}$ would be

$$
\frac{1}{2} \mathfrak{C}_{0}^{(M N)(R S)(A B)} \delta_{\mathcal{B}} g_{A B} \delta_{\mathcal{B}} g_{R S}
$$

However, we would get the same contribution to $T^{M N}$ if we started with a $(R S) \leftrightarrow(A B)$ swapped term $\mathfrak{C}_{0}^{(M N)(A B)(R S)} \delta_{\mathcal{B}} g_{A B} \in \mathfrak{C}_{0}^{(M N)(R S)}$ instead. Therefore to avoid overcounting, we need to ensure that if we include a term $\mathfrak{c}_{0}^{(M N)(R S)(A B)} \delta_{\mathcal{B}} g_{A B}$ in $\mathfrak{c}_{0}^{(M N)(R S)}$, we should drop out a corresponding term $\mathfrak{夭}_{0}^{(M N)(A B)(R S)} \delta_{\mathcal{B}} g_{A B}$ which would give the same contribution to the constitutive relations. Keeping in mind this minor technicality, we have enlisted all the possible terms in $\mathfrak{夭}_{0}$ and their contribution to $T^{M N}$ in Table IV. There is no associated free energy current.

Let us now move on to $\mathfrak{C}_{1}$. We need to write down the most generic 5-tensor $\mathfrak{C}_{1}^{(M N)(R S) T}$ made out of 0-derivative data. Note that the index $T$ cannot come from a $V^{T}$ because the respective term will have a contraction with $\nabla_{T}$ causing it to vanish. The remaining 4 indices cannot come from a $u^{M}$ as the respective terms have been eliminated using the equations of motion. The resultant allowed terms in $\mathfrak{C}_{1}$ have been enlisted in Table $\mathrm{V}$ along with their contribution to the constitutive relations. The respective contribution to the free energy current has been given in Table VI.

It should be noted that there is a plausible term $\alpha V^{(M} P^{N)(R} V^{S)} u^{T}$ in $\mathfrak{S}_{1}$ which we have not included in Table V. Its contribution to $T^{M N}$, after some simplification, would have been

$$
\begin{aligned}
- & \frac{1}{2} V^{(M}\left[\alpha \nabla^{N)}\left(\frac{1}{T} \Theta_{T}\right)-\frac{1}{T^{2}} \alpha \nabla^{N)} u^{R} \nabla_{R} T+\frac{1}{2 T} \alpha \tau^{N)} \Theta\right. \\
& \left.+\frac{1}{2 T} \tau^{N)} u^{R} \partial_{R} \alpha\right] .
\end{aligned}
$$

Note that the last three terms are composites and are linearly dependent on the contributions from $\mathfrak{C}_{0}$. The first

TABLE V. Zero derivative terms in $\mathfrak{夭}_{1}^{(M N)(R S) T}$ and their contribution to the second order Class D and $\overline{\mathrm{D}}$ constitutive relations. Here we have defined $\delta_{i}^{ \pm}=\left(\delta_{i} \pm \bar{\delta}_{i}\right) / 2$. The transport coefficients $\delta_{i}$ couple to Class D constitutive relations while $\bar{\delta}_{i}$ couples to Class $\overline{\mathrm{D}}$.

\begin{tabular}{lcc}
\hline \hline Coefficient & $\mathfrak{G}_{1}^{(M N)(R S) T}$ & $T^{M N} \ni-\mathfrak{c}_{1}^{(M N)(R S) T} \frac{1}{2} \nabla_{T} \delta_{\mathcal{B}} g_{R S}-\frac{1}{2} \nabla_{T} \mathfrak{G}_{1}^{(M N)(R S) T} \frac{1}{2} \delta_{\mathcal{B}} g_{R S}$ \\
\hline$\delta_{10}^{+}$ & $2 T \delta_{10}^{+} P^{T(M} V^{N)} P^{R S}$ & $-2 V^{(M} P^{N) R}\left[\delta_{10}^{+} \partial_{R} \Theta+\frac{T}{2} \Theta \partial_{R}\left(\frac{\delta_{10}^{+}}{T}\right)\right]+\delta_{10}^{+} V^{(M} \nabla^{N)} u_{R} \tau^{R}$ \\
$\delta_{10}^{-}$ & $-2 T \delta_{10}^{-} P^{M N} V^{(R} P^{S) T}$ & $P^{M N}\left[\delta_{10}^{-} \nabla_{R} \tau^{R}+\frac{T}{2} \tau^{R} \partial_{R}\left(\frac{\delta_{10}^{-1}}{T}\right)\right]+\delta_{10}^{-} V^{(M} \nabla_{R} u^{N)} \tau^{R}$ \\
$\delta_{11}^{+}$ & $-V^{(M} P^{N)}\left[\delta_{11}^{+} \nabla_{T} \sigma^{R T}+\frac{T}{2} \sigma^{R T} \partial_{T}\left(\frac{\delta_{11}^{+}}{T}\right)\right]$ \\
& $2 T \delta_{11}^{+} V^{(M} P^{N)\langle R} P^{S\rangle T}$ & $+\frac{1}{2} \delta_{11}^{+} V^{(M}\left(\nabla_{R} u^{N)}+P^{N)}{ }_{R} \Theta-\frac{2}{d} \nabla^{N)} u_{R}\right) \tau^{R}$ \\
$\delta_{11}^{-}$ & & $\left.P^{T\langle M} P^{N\rangle}{ }_{R}\left[\delta_{11}^{-} \nabla_{T} \tau^{R}+\frac{T}{2} \tau^{R} \partial_{T} \frac{\delta_{11}^{-1}}{T}\right)\right]$ \\
& $-2 T \delta_{11}^{-} P^{T\langle M} P^{N\rangle(R} V^{S)}$ & $+\frac{1}{2} \delta_{11}^{-} V^{(M}\left(\nabla^{N)} u_{R}+P^{N)}{ }_{R} \Theta-\frac{2}{d} \nabla_{R} u^{N)}\right) \tau^{R}$ \\
$\bar{\delta}_{12}$ & $2 T \bar{\delta}_{12} P^{M N} P^{R S} u^{T}$ & $-2 P^{M N}\left[\bar{\delta}_{12} u^{R} \partial_{R} \Theta+\frac{T}{2} \Theta u^{R} \partial_{R}\left(\frac{\bar{\delta}_{12}}{T}\right)\right]$ \\
& & $-P^{M N} \bar{\delta}_{12}\left(\Theta^{2}-a^{R} \tau_{R}\right)-2 \bar{\delta}_{12} V^{(M} a^{N)} \Theta$ \\
$\bar{\delta}_{13}$ & $2 T \bar{\delta}_{13} P^{M\langle R} P^{S\rangle N} u^{T}$ & $\left.\left.-P^{M}{ }_{R} P^{N}{ }_{S} \bar{\delta}_{13} u^{R} \nabla_{R} \sigma^{R S}+\frac{T}{2} \sigma^{R S} u^{T} \partial_{T} \bar{\delta}_{13}^{T}\right)\right]$ \\
& & $-\bar{\delta}_{13}\left(\frac{1}{2} \sigma^{M N} \Theta-a^{\langle M} \tau^{N\rangle}\right)-\bar{\delta}_{13} V^{(M} \sigma^{N) R} a_{R}$ \\
\hline \hline
\end{tabular}


TABLE VI. Class $\bar{D}$ free energy current up to second derivative order. This is the only contribution to the nonhydrostatic free energy current.

\begin{tabular}{lc}
\hline \hline Coefficient & $N^{M} \in-\frac{1}{8} \mathfrak{c}_{1}^{(R S)(A B) M} \delta_{\mathcal{B}} g_{R S} \delta_{\mathcal{B}} g_{A B}$ \\
\hline $\bar{\delta}_{10}$ & $-\frac{\bar{\delta}_{10}}{2 T} \tau^{M} \Theta$ \\
$\bar{\delta}_{11}$ & $-\frac{\bar{\delta}_{11}}{4 T} \sigma^{M N} \tau_{N}$ \\
$\bar{\delta}_{12}$ & $-\frac{\bar{\delta}_{12}}{T} u^{M} \Theta^{2}$ \\
$\bar{\delta}_{13}$ & $-\frac{\bar{\delta}_{13}}{4 T} u^{M} \sigma^{M N} \sigma_{M N}$ \\
\hline \hline
\end{tabular}

term is pure derivative, but is made linearly dependent by the equations of motion. Hence the contribution of $\alpha V^{(M} P^{N)(R} V^{S)} u^{T}$ is not linearly independent onshell. It is worth pointing out that this apparent exception is another "unnatural" consequence of working with no torsion. In presence of torsion, the respective contribution would have included an independent term coupling to $V^{(M} u^{R} \nabla_{R} \tau^{N)}$.

\section{Mass frame}

In our analysis above, we had fixed the hydrodynamic redefinition freedom in $u^{M}, T$ and $\mu_{m}$ by relating them to $\beta^{M}$, as defined in Eq. (10). Although this is a convenient choice of frame for computations, it is not the most physically interesting one. A better physically motivated choice is the so called "mass frame," in which the mass current (after null reduction) does not get any derivative corrections. It is defined as

$$
T_{\mathrm{mf}}^{M N} V_{N}=-R u^{M}-E V^{M} .
$$

Let us start with the results in our frame and schematically represent them as

$$
\begin{aligned}
T^{M N}= & R u^{M} u^{N}+2 E u^{(M} V^{N)}+P P^{M N}+\mathcal{T}^{M N} \\
& +\mathcal{O}\left(\partial^{3}\right),
\end{aligned}
$$

where the tensor $\mathcal{T}^{M N}$ contains all the derivative corrections. A generic change of frame would amount to a field redefinition

$u^{M} \rightarrow u^{M}+\delta u^{M}, \quad T \rightarrow u^{M}+\delta T, \quad \varpi \rightarrow u^{M}+\delta \varpi$.

We can check that the first order null fluid automatically respects the mass frame, so we only need to perform this redefinition for second order variations $\delta u^{M}, \delta T$, and $\delta \varsigma$. This immediately implies that $\mathcal{T}^{M N}$ remains unchanged up to three derivative terms. Under a second order redefinition therefore, the energy momentum tensor changes as

$$
\begin{aligned}
T_{\mathrm{mf}}^{M N}= & R u^{M} u^{N}+2 E u^{(M} V^{N)}+P P^{M N}+2 R u^{(M} \delta u^{N)} \\
& +\left(\frac{\partial R}{\partial T} \delta T+\frac{\partial R}{\partial \varpi} \delta \varpi\right) u^{M} u^{N}+2(E+P) V^{(M} \delta u^{N)} \\
& +2\left(\frac{\partial E}{\partial T} \delta T+\frac{\partial E}{\partial \varpi} \delta \varpi\right) u^{(M} V^{N)} \\
& +\left(\frac{E+P}{T} \delta T+T R \delta \varpi\right) P^{M N} \\
& +\mathcal{T}^{M N}+\mathcal{O}\left(\partial^{3}\right) .
\end{aligned}
$$

The condition (44) then requires

$$
\begin{aligned}
\left(\begin{array}{c}
\frac{1}{T} \delta T \\
T \delta \varpi
\end{array}\right) & =-\left(\begin{array}{ll}
T \frac{\partial E}{\partial T} & \frac{1}{T} \frac{\partial E}{\partial \varpi} \\
T \frac{\partial R}{\partial T} & \frac{1}{T} \frac{\partial R}{\partial \varpi}
\end{array}\right)^{-1}\left(\begin{array}{c}
\mathcal{T}^{M N} u_{M} V_{N} \\
\mathcal{T}^{M N} V_{M} V_{N}
\end{array}\right), \\
\delta u^{M} & =\frac{1}{R} P^{M}{ }_{N} \mathcal{T}^{N R} V_{R} .
\end{aligned}
$$

After plugging these back into Eq. (47), we can get $T^{M N}$ in the mass frame

$$
\begin{aligned}
T_{\mathrm{mf}}^{M N}= & R u^{M} u^{N}+2 E u^{(M} V^{N)}+P P^{M N}+\mathcal{T}_{\mathrm{mf}}^{M N} \\
& +\mathcal{O}\left(\partial^{3}\right),
\end{aligned}
$$

where

$$
\begin{aligned}
\mathcal{T}_{\mathrm{mf}}^{M N}= & 2 V^{(M} P^{N)}{ }_{R} \mathcal{T}^{R S}\left(\frac{E+P}{R} V_{S}-u_{S}\right) \\
& -\mathcal{T}^{R S}\left(\frac{\partial P}{\partial E} u_{R} V_{S}+\frac{\partial P}{\partial R} V_{R} V_{S}\right) P^{M N} \\
& +P^{M}{ }_{R} P^{N}{ }_{S} \mathcal{T}^{M N}
\end{aligned}
$$

This finishes our discussion of second order null fluids. The respective constitutive relations and free energy current, in off-shell hydrodynamic frame, have been summarized in Tables II-VI. If the reader is instead interested in the results in mass frame, the formula for translation is given in Eq. (50). In the next section, we will perform null reduction on these results to obtain the constitutive relations of a second order Galilean fluid.

\section{SECOND ORDER GALILEAN FLUIDS VIA NULL REDUCTION}

We will now reduce our null fluid results presented in Sec. III, to obtain the constitutive relations of a Galilean fluid up to second order. We will mainly focus on the covariant Newton-Cartan notation to deal with Galilean fluids coupled to curved backgrounds. Later, specializing to flat backgrounds, we will also discuss the conversion of these results to the conventional noncovariant notation. For more details, please refer to our previous work [11]. 


\section{A. Newton-Cartan backgrounds}

Newton-Cartan geometries are a covariant representation of spacetimes which respect Galilean symmetries. As we established in [11], Newton-Cartan backgrounds are related to null backgrounds by a mere choice of basis. In the following we briefly review the argument, and in the process introduce the reader to the basics of NewtonCartan backgrounds. For a fuller and excellent review of Newton-Cartan geometries, please refer to the Appendix of [28].

On a null background, we choose a basis $\left\{x^{M}\right\}=\left\{x^{-}, x^{\mu}\right\}$ such that the null isometry $\mathcal{V}=\left\{V=\partial_{-}\right\}$. The fact that $\mathcal{V}$ is an isometry implies that all the fields in the theory are independent of the $x^{-}$coordinate. To perform the reduction, we require an arbitrary null field $v^{M}$ normalized as $v^{M} v_{M}=0, v^{M} V_{M}=-1$, which can be interpreted as providing a "Galilean frame of reference." In the case of a null fluid, the null fluid velocity $v^{M}=u^{M}$ defines a special Galilean frame which we refer to as the "fluid frame of reference." In an arbitrary Galilean frame, we decompose the fields $V^{M}, v^{M}$, and $g_{M N}$ in the chosen basis as

$$
\begin{aligned}
& V^{M}=\left(\begin{array}{l}
1 \\
0
\end{array}\right), \quad v^{M}=\left(\begin{array}{c}
v^{\mu} B_{\mu}^{(v)} \\
v^{\mu}
\end{array}\right), \\
& g_{M N}=\left(\begin{array}{cc}
0 & -n_{\nu} \\
-n_{\mu} & h_{\mu \nu}+2 n_{(\mu} B_{\nu)}^{(v)}
\end{array}\right) \text {, }
\end{aligned}
$$

along with

$$
\begin{aligned}
V_{M} & =\left(\begin{array}{c}
0 \\
-n_{\mu}
\end{array}\right), \quad v_{M}=\left(\begin{array}{c}
-1 \\
B_{\mu}^{(v)}
\end{array}\right), \\
g^{M N} & =\left(\begin{array}{cc}
h^{\nu \rho} B_{\nu}^{(v)} B_{\rho}^{(v)}-2 v^{\mu} B_{\mu}^{(v)} & h^{\nu \rho} B_{\rho}^{(v)}-v^{\nu} \\
h^{\mu \nu} B_{\nu}^{(v)}-v^{\mu} & h^{\mu \nu}
\end{array}\right),
\end{aligned}
$$

such that

$$
\begin{aligned}
n_{\mu} v^{\mu} & =1, & & v^{\mu} h_{\mu \nu}=0, \\
n_{\mu} h^{\mu \nu} & =0, & & h_{\mu \rho} h^{\rho \nu}+n_{\mu} v^{\nu}=\delta_{\mu}{ }^{\nu} .
\end{aligned}
$$

The collection of fields $\left\{n_{\mu}, v^{\mu}, h^{\mu \nu}, h_{\mu \nu}, B_{\mu}^{(v)}\right\}$ defines a Newton-Cartan structure. The torsionlessness condition $H_{M N}=0$ implies that the "time-metric" $n=n_{\mu} \mathrm{d} x^{\mu}$ is a closed one-form, i.e., $\mathrm{d} n=0$; this is known to be true for torsionless Newton-Cartan structures. Note that after choosing the said basis, the residual diffeomorphisms are $x^{\mu} \rightarrow x^{\mu}+\chi^{\mu}\left(x^{\nu}\right)$ and $x^{-} \rightarrow \xi^{-}+\chi^{-}\left(x^{\mu}\right)$. The former of these are just the Newton-Cartan diffeomorphisms, while the latter are known as "mass gauge transformations." $B_{\mu}^{(v)}$ is the only field that transforms under this gauge transformation

$$
\delta_{\chi^{-}} B_{\mu}^{(v)}=-\partial_{\mu} \chi^{-},
$$

and hence is known as the mass gauge field. The LeviCivita connection $\Gamma^{R}{ }_{M N}$ decomposes in this basis as

$$
\begin{aligned}
\Gamma_{\mu \nu}^{\lambda}= & v^{\lambda} \partial_{(\mu} n_{\nu)}+\frac{1}{2} h^{\lambda \rho}\left(\partial_{\mu} h_{\rho \nu}+\partial_{\nu} h_{\rho \mu}-\partial_{\rho} h_{\mu \nu}\right) \\
& -\Omega_{\sigma(\mu}^{(v)} n_{\nu)} h^{\sigma \lambda}, \\
\Gamma_{\mu \nu}^{-}= & h_{\lambda(\mu} \tilde{\nabla}_{\nu)} v^{\lambda}-\tilde{\nabla}_{(\mu} B_{\nu)}^{(v)},
\end{aligned}
$$

and all the remaining components zero. Here we have identified $\Gamma_{\mu \nu}^{\lambda}$ as the (torsionless) Newton-Cartan connection and denoted the respective covariant derivative by $\tilde{\nabla}_{\mu}$. We have also defined the (dual) frame vorticity as

$$
\Omega_{\mu \nu}^{(v)}=2 h_{\sigma[\nu} \tilde{\nabla}_{\mu]} v^{\sigma}=\partial_{\mu} B_{\nu}^{(v)}-\partial_{\nu} B_{\mu}^{(v)} .
$$

The covariant derivative $\tilde{\nabla}$ acts on the Newton-Cartan structure appropriately

$$
\begin{aligned}
\tilde{\nabla}_{\mu} n_{\nu} & =0, \quad \tilde{\nabla}_{\mu} h^{\rho \sigma}=0, \\
\tilde{\nabla}_{\mu} h_{\nu \rho} & =-2 n_{(\nu} h_{\rho) \sigma} \tilde{\nabla}_{\mu} v^{\sigma} .
\end{aligned}
$$

Note that $v^{M}$ was an arbitrary field chosen to perform the reduction, and one is allowed to arbitrarily redefine it without changing the physics. This leads to the invariance of the system under "Milne transformations" of the Newton-Cartan structure

$$
\begin{aligned}
v^{\mu} & \rightarrow v^{\mu}+\psi^{\mu}, \quad h_{\mu \nu} \rightarrow h_{\mu \nu}-2 n_{(\mu} \psi_{\nu)}+n_{\mu} n_{\nu} \psi^{\rho} \psi_{\rho}, \\
B_{\mu}^{(v)} & \rightarrow B_{\mu}^{(v)}+\psi_{\mu}-\frac{1}{2} n_{\mu} \psi^{\rho} \psi_{\rho},
\end{aligned}
$$

where $\psi^{\mu} n_{\mu}=0, \psi_{\mu}=h_{\mu \nu} \psi^{\nu}$. The fields $n_{\mu}, h^{\mu \nu}$ and the connection $\Gamma_{\mu \nu}^{\rho}$ on the other hand are Milne invariant.

We can now decompose the fluid velocity $u^{M}$ and the associated projector $P^{M N}$ as

$$
\begin{aligned}
& u^{M}=\left(\begin{array}{c}
u^{\mu} B_{\mu} \\
u^{\mu}
\end{array}\right), \quad u_{M}=\left(\begin{array}{c}
-1 \\
B_{\mu}
\end{array}\right), \\
& P_{M N}=\left(\begin{array}{cc}
0 & 0 \\
0 & p_{\mu \nu}
\end{array}\right), \quad P^{M N}=\left(\begin{array}{cc}
p^{\nu \rho} B_{\nu} B_{\rho} & p^{\mu \nu} B_{\nu} \\
p^{\mu \nu} B_{\nu} & p^{\mu \nu}
\end{array}\right) .
\end{aligned}
$$

The fields $\left\{n_{\mu}, u^{\mu}, p^{\mu \nu}, p_{\mu \nu}, B_{\mu}\right\}$ define the Newton-Cartan structure in the fluid frame of reference, satisfying,

$$
\begin{aligned}
n_{\mu} u^{\mu} & =1, & & u^{\mu} p_{\mu \nu}=0, \\
n_{\mu} p^{\mu \nu} & =0, & & p_{\mu \rho} p^{\rho \nu}+n_{\mu} u^{\nu}=\delta_{\mu}{ }^{\nu} .
\end{aligned}
$$


They can be reexpressed in terms of $\left\{n_{\mu}, v^{\mu}, h^{\mu \nu}, h_{\mu \nu}, B_{\mu}^{(v)}\right\}$ using Eq. (58) with $\psi^{\mu}=\bar{u}^{\mu}=h^{\mu}{ }_{\nu} u^{\nu}=u^{\mu}-v^{\mu}$,

$$
\begin{aligned}
p^{\mu \nu} & =h^{\mu \nu}, \quad p_{\mu \nu}=h_{\mu \nu}-2 n_{(\mu} \bar{u}_{\nu)}+n_{\mu} n_{\nu} \bar{u}^{\rho} \bar{u}_{\rho} \\
B_{\mu} & =B_{\mu}^{(v)}+\bar{u}_{\mu}-\frac{1}{2} n_{\mu} \bar{u}^{\rho} \bar{u}_{\rho} .
\end{aligned}
$$

The (dual) fluid vorticity is defined similar to the (dual) frame vorticity as,

$$
\Omega_{\mu \nu}=2 p_{\sigma[\nu} \tilde{\nabla}_{\mu]} u^{\sigma}=\partial_{\mu} B_{\nu}-\partial_{\nu} B_{\mu}
$$

The mass current $\rho^{\mu}$, energy current $\epsilon^{\mu}$, and stress tensor $t^{\mu \nu}$ on Newton-Cartan backgrounds can be respectively read out in terms of $T^{M N}$ as (see [11] for details)

$\rho^{\mu}=-T^{\mu M} V_{M}, \quad \epsilon^{\mu}=-T^{\mu M} u_{M}, \quad t^{\mu \nu}=P^{\mu}{ }_{M} P^{\nu}{ }_{N} T^{M N}$.

with $t^{\mu \nu}=t^{\nu \mu}$ and $t^{\mu \nu} n_{\nu}=0$. They satisfy the conservation laws

Mass Conservation: $\tilde{\nabla}_{\mu} \rho^{\mu}=0$,

Energy Conservation: $\tilde{\nabla}_{\mu} \epsilon^{\mu}=-\left(u^{\mu} \rho^{\sigma}+t^{\mu \sigma}\right) p_{\sigma \nu} \tilde{\nabla}_{\mu} u^{\nu}$,

Momentum Conservation: $\tilde{\nabla}_{\mu}\left(u^{\mu} p^{\sigma}{ }_{\nu} \rho^{\nu}+t^{\mu \sigma}\right)=-\rho^{\mu} \tilde{\nabla}_{\mu} u^{\sigma}$.

Here, the energy current $\epsilon^{\mu}$ and the stress tensor $t^{\mu \nu}$ in Eq. (63) are defined in the fluid frame of reference; we can define the respective quantities in an arbitrary frame of reference as

$$
\begin{aligned}
& \epsilon_{(v)}^{\mu}=-T^{\mu M} v_{M}=\epsilon^{\mu}+u^{\mu} \bar{u}^{\nu} p_{\nu \rho} \rho^{\rho}+\frac{1}{2} \rho^{\mu} \bar{u}^{\rho} \bar{u}_{\rho}+t^{\mu \nu} \bar{u}_{\nu}, \\
& t_{(v)}^{\mu \nu}=\left(P_{(v)}\right)^{\mu}{ }_{M}\left(P_{(v)}\right)_{N}{ }_{N} T^{M N}=t^{\mu \nu}+2 \bar{u}^{(\mu} h^{\nu)}{ }_{\sigma} \rho^{\sigma}-\bar{u}^{\mu} \bar{u}^{\nu} \rho^{\sigma} n_{\sigma},
\end{aligned}
$$

where $P_{(v)}^{M N}=g^{M N}+2 v^{(M} V^{N)}$. They satisfy the respective conservation laws

$$
\begin{aligned}
& \tilde{\nabla}_{\mu} \epsilon_{(v)}^{\mu}=-\left(v^{\mu} \rho^{\sigma}+t_{(v)}^{\mu \sigma}\right) h_{\sigma \nu} \tilde{\nabla}_{\mu} v^{\nu} \\
& \tilde{\nabla}_{\mu}\left(v^{\mu} h^{\sigma}{ }_{\nu} \rho^{\nu}+t_{(v)}^{\mu \sigma}\right)=-\rho^{\mu} \tilde{\nabla}_{\mu} v^{\sigma} .
\end{aligned}
$$

In the following, we will only present the constitutive relations in the fluid frame of reference. However, we can always use Eq. (65) to go to any arbitrary frame.

\section{B. Second order Galilean fluids}

Having reviewed the general rules of null reduction, we can now go on and reduce the null fluid constitutive relations. To setup the notation, we have reduced the first order fluid data in Table VII. We can use the formulas in Eq. (63) to convert the null fluid energy momentum tensor $T^{M N}$ in Tables II, IV, and V into Galilean fluid mass current $\rho^{\mu}$ in Table VIII, energy current $\epsilon^{\mu}$ in Table IX and stress tensor stress tensor $t^{\mu \nu}$ in Table X. The results schematically look like

$\rho^{\mu}=\left(R+\varsigma_{\rho}\right) u^{\mu}+\varsigma_{\rho}^{\mu}, \quad \epsilon^{\mu}=\left(E+\varsigma_{\epsilon}\right) u^{\mu}+\varsigma_{\epsilon}^{\mu}$,

$t^{\mu \nu}=P p^{\mu \nu}+\varsigma_{t}^{\mu \nu}$.

The tensors $\varsigma_{\rho}, \varsigma_{\rho}^{\mu}, \varsigma_{\epsilon}, \varsigma_{\epsilon}^{\mu}$ and $\varsigma_{t}^{\mu \nu}$ contain derivative corrections with $\varsigma_{t}^{\mu \nu}=\varsigma_{t}^{\nu \mu}$ and $\varsigma_{\rho}^{\mu} n_{\mu}=\varsigma_{\epsilon}^{\mu} n_{\mu}=\varsigma_{t}^{\mu \nu} n_{\mu}=0$. These are the Galilean fluid constitutive relations in the offshell hydrodynamic frame. If we are rather interested in

TABLE VII. First order fluid data in null background, Newton-Cartan and noncovariant notations. Noncovariant results have been specialized to flat backgrounds.

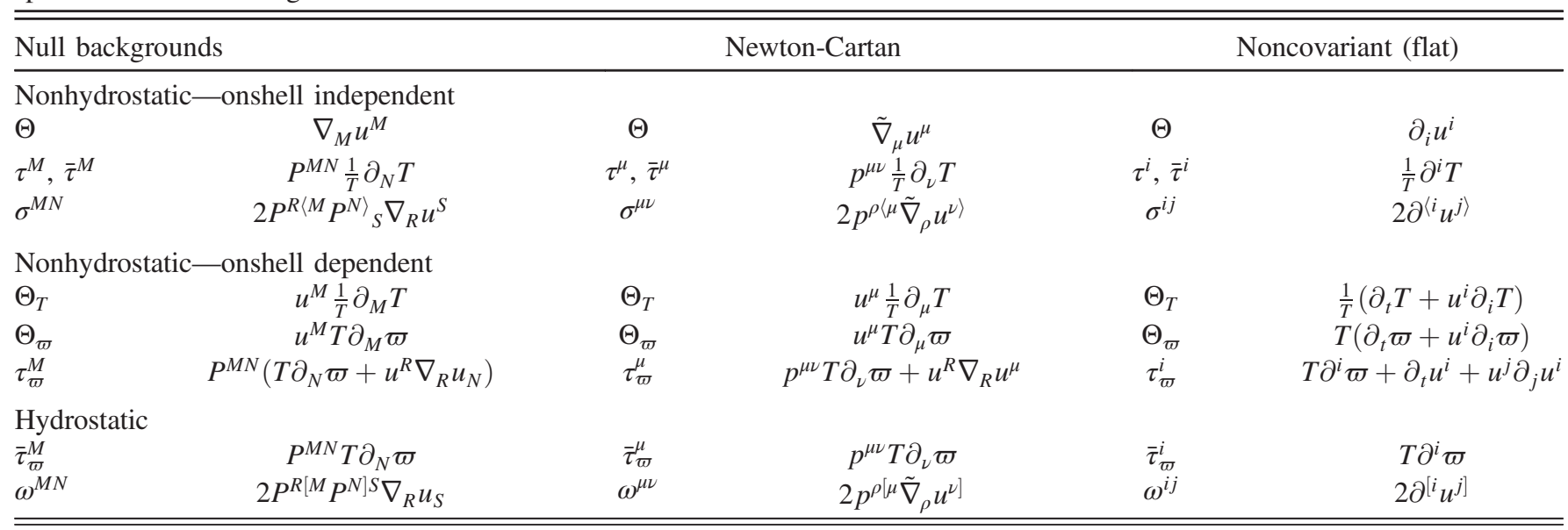


TABLE VIII. Mass current of a Galilean fluid up to second derivative order in off-shell frame and mass frame respectively. Note that in mass frame, the mass current is simply $\rho_{\mathrm{mf}}^{\mu}=R u^{\mu}$.

\begin{tabular}{lcc}
\hline \hline Coefficient & $\rho^{\mu}$ & $\rho_{\mathrm{mf}}^{\mu}$ \\
\hline$P$ & $\frac{1}{T} \frac{\partial P}{\partial \varpi} u^{\mu} \equiv R u^{\mu}$ & \\
$\alpha_{1}$ & $\frac{1}{T}\left(\frac{1}{2} \frac{\partial \alpha_{1}}{\partial \varpi} \bar{\tau}_{\varpi}^{\mu} \bar{\tau}_{\varpi \mu}-\tilde{\nabla}_{\nu}\left(\alpha_{1} T \bar{\tau}_{\varpi}^{\nu}\right)\right) u^{\mu}$ & 0 \\
$\alpha_{2}$ & $\frac{1}{T}\left(\frac{\partial \alpha_{2}}{\partial \varpi} \bar{\tau}_{\varpi}^{\mu} \bar{\tau}_{\mu}-\tilde{\nabla}_{\nu}\left(\alpha_{2} T \bar{\tau}^{\nu}\right)\right) u^{\mu}$ & 0 \\
$\alpha_{3}$ & $\frac{1}{4 T} \frac{\partial \alpha_{3}}{\partial \varpi} \omega^{\rho \sigma} \omega_{\rho \sigma} u^{\mu}-\tilde{\nabla}_{\nu}\left(\alpha_{3} \omega^{\nu \mu}\right)$ & 0 \\
$\alpha_{5}$ & $\frac{1}{T} \frac{\partial \alpha_{5}}{\partial \varpi} R u^{\mu}$ & 0 \\
\hline \hline
\end{tabular}

the results in mass frame defined in Sec. III C, we can reduce Eq. (50) to get

$\varsigma_{\rho, \mathrm{mf}}=\varsigma_{\rho, \mathrm{mf}}^{\mu}=\varsigma_{\epsilon, \mathrm{mf}}=0, \quad \varsigma_{\epsilon, \mathrm{mf}}^{\mu}=\varsigma_{\epsilon}^{\mu}-\frac{E+P}{R} \varsigma_{\rho}^{\mu}$,

$\varsigma_{t, \mathrm{mf}}^{\mu \nu}=\varsigma_{t}^{\mu \nu}-p^{\mu \nu}\left(\frac{\partial P}{\partial E} \varsigma_{\epsilon}+\frac{\partial P}{\partial R} \varsigma_{\rho}\right)$.

These mass frame results have also been presented in Tables VIII-X alongside their offshell frame counterparts.
Summary of transport coefficients: At ideal order, there is just one independent transport coefficient: the thermodynamic pressure $P$. Thermodynamic energy density $E$ and mass density $R$ are determined in terms of $P$ via the thermodynamic relation

$$
\mathrm{d} P=\frac{E+P}{T} \mathrm{~d} T+T R \mathrm{~d} \varpi .
$$

At first order, there are three new transport coefficients: shear viscosity $\eta$, bulk viscosity $\zeta$ and thermal conductivity $\kappa$. All three of them are dissipative and are required to be non-negative by the second law. At second order, there are 25 independent transport coefficients. 5 of them are hydrostatic belonging to Class $\mathrm{H}_{\mathrm{S}}$

$$
\alpha_{1}, \quad \alpha_{2}, \quad \alpha_{3}, \quad \alpha_{4}, \quad \alpha_{5}
$$

Other 20 are nonhydrostatic. 11 in Class $\overline{\mathrm{D}}$

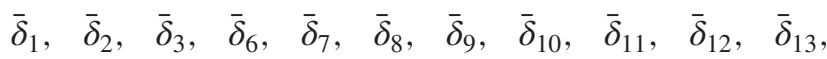

TABLE IX. Energy current of a Galilean fluid up to second derivative order in off-shell frame and mass frame

\begin{tabular}{|c|c|c|}
\hline Coefficient & $\epsilon^{\mu}$ & $\epsilon_{\mathrm{mf}}^{\mu}$ \\
\hline$P$ & \multicolumn{2}{|c|}{$T^{2} \frac{\partial(P / T)}{\partial T} u^{\mu} \equiv E u^{\mu}$} \\
\hline$\kappa T$ & \multicolumn{2}{|c|}{$-T \kappa \tau^{\mu}$} \\
\hline$\alpha_{1}$ & $\frac{1}{2} \frac{\partial\left(T \alpha_{1}\right)}{\partial T} \bar{\tau}_{\varpi}^{\rho} \bar{\tau}_{\varpi \rho} u^{\mu}+\alpha_{1} \bar{\tau}_{\varpi}^{\mu} \Theta_{\varpi}$ & $\alpha_{1} \bar{\tau}_{\varpi}^{\mu} \Theta_{\varpi}$ \\
\hline$\alpha_{2}$ & $\begin{array}{l}{\left[T^{2} \frac{\partial\left(\alpha_{2} / T\right)}{\partial T} \bar{\tau}_{\varpi}^{\rho} \bar{\tau}_{\rho}-T \nabla_{\nu}\left(\frac{\alpha_{2}}{T} \bar{\tau}_{\varpi}^{\nu}\right)\right] u^{\mu}} \\
\quad+\alpha_{2} \bar{\tau}^{\mu} \Theta_{\varpi}+\alpha_{2} \bar{\tau}_{\varpi}^{\mu} \Theta_{T}\end{array}$ & $\alpha_{2} \bar{\tau}^{\mu} \Theta_{\varpi}+\alpha_{2} \bar{\tau}_{\varpi}^{\mu} \Theta_{T}$ \\
\hline$\alpha_{3}$ & $\frac{1}{4} T^{2} \frac{\partial\left(\alpha_{3} / T\right)}{\partial T} \omega^{\nu \rho} \omega_{\nu \rho} u^{\mu}+\alpha_{3} \omega^{\mu \nu} a_{\nu}$ & $\alpha_{3} \omega^{\mu \nu} a_{\nu}+\frac{E+P}{R} \tilde{\nabla}_{\nu}\left(\alpha_{3} \omega^{\nu \mu}\right)$ \\
\hline$\alpha_{4}$ & $-\tilde{\nabla}_{\nu}\left(\alpha_{4} \omega^{\nu \mu}\right)$ & $-\tilde{\nabla}_{\nu}\left(\alpha_{4} \omega^{\nu \mu}\right)$ \\
\hline$\alpha_{5}$ & $\begin{array}{l}\left(T^{2} \frac{\partial\left(\alpha_{5} / T\right)}{\partial T} R+2 p^{\rho \sigma} \tilde{\nabla}_{\rho} \tilde{\nabla}_{\sigma} \alpha_{5}\right) u^{\mu} \\
+2 \alpha_{5} p^{\mu \nu} R_{\nu \rho} u^{\rho}-2 p^{\mu \nu} u^{\rho} \tilde{\nabla}_{\nu} \tilde{\nabla}_{\rho} \alpha_{5}\end{array}$ & $2 \alpha_{5} p^{\mu \nu} R_{\nu \rho} u^{\rho}-2 p^{\mu \nu} u^{\rho} \tilde{\nabla}_{\nu} \tilde{\nabla}_{\rho} \alpha_{5}$ \\
\hline $\begin{array}{l}\delta_{1}^{+} \\
\delta_{2}^{+}\end{array}$ & \multicolumn{2}{|c|}{$-\frac{1}{2} \delta_{1}^{+} \sigma^{\mu \nu} \tau_{\nu}$} \\
\hline $\begin{array}{l}\delta_{2} \\
\delta_{6}^{+}\end{array}$ & $-\frac{1}{2} \delta_{6}^{+} \sigma^{\mu \nu} \bar{\tau}_{\varpi \nu}$ & $-\delta_{2}^{+} \tau^{\mu} \Theta$ \\
\hline$\delta_{7}^{+}$ & \multicolumn{2}{|c|}{$-\delta_{7}^{+} \bar{\tau}_{\varpi}^{\mu} \Theta$} \\
\hline $\bar{\delta}_{8}$ & \multicolumn{2}{|c|}{$-\frac{1}{2} \bar{\delta}_{8} \omega^{\mu \rho} \tau_{\rho}$} \\
\hline$\delta_{10}^{+}$ & \multicolumn{2}{|c|}{$-p^{\mu \rho}\left[\delta_{10}^{+} \partial_{\rho} \Theta+\frac{T}{2} \Theta \partial_{\rho}\left(\frac{\delta_{10}^{+}}{T}\right)\right]+\frac{1}{2} \delta_{10}^{+} p^{\mu \nu} \tau_{\sigma} \tilde{\nabla}_{\nu} u^{\sigma}$} \\
\hline$\delta_{10}^{-}$ & \multicolumn{2}{|c|}{$-\frac{1}{2} \delta_{10}^{-} \tau^{\sigma} \tilde{\nabla}_{\sigma} u^{\mu}$} \\
\hline$\delta_{11}^{+}$ & \multirow{3}{*}{\multicolumn{2}{|c|}{$\begin{array}{c}-\frac{1}{2}\left[\delta_{11}^{+} \tilde{\nabla}_{\nu} \sigma^{\mu \nu}+\frac{T}{2} \sigma^{\mu \nu} \partial_{\nu}\left(\frac{\delta_{11}^{+}}{T}\right)\right]+\frac{1}{4} \delta_{11}^{+}\left(\tau^{\nu} \tilde{\nabla}_{\nu} u^{\mu}+\tau^{\mu} \Theta-\frac{2}{d} p^{\mu \rho} \tau_{\sigma} \tilde{\nabla}_{\rho} u^{\sigma}\right) \\
-\frac{1}{4} \delta_{11}^{-}\left(p^{\mu \rho} \tau_{\sigma} \tilde{\nabla}_{\rho} u^{\sigma}+\tau^{\mu} \Theta-\frac{2}{d} \tau^{\nu} \tilde{\nabla}_{\nu} u^{\mu}\right) \\
-\bar{\delta}_{12} a^{\mu} \Theta \\
-\frac{1}{2} \bar{\delta}_{13} \sigma^{\mu \nu} a_{\nu}\end{array}$}} \\
\hline$\delta_{11}^{-}$ & & \\
\hline $\begin{array}{l}\bar{\delta}_{12} \\
\bar{\delta}_{13}\end{array}$ & & \\
\hline
\end{tabular}
respectively. Note that the only difference in the two frames comes in the hydrostatic sector. 
$\delta_{1}, \quad \delta_{2}, \quad \delta_{3}, \quad \delta_{4}, \quad \delta_{5}, \quad \delta_{6}, \quad \delta_{7}, \quad \delta_{10}, \quad \delta_{11}$.

Second law gives no inequality constraints on these transport coefficients. For some transport coefficients in Tables VIII-X, we have denoted $\delta_{i}^{ \pm}=\left(\delta \pm \bar{\delta}_{i}\right) / 2$.

\section{Noncovariant results in flat spacetime}

When the Galilean fluid is coupled to a flat background, it is perhaps more fitting to express the results in the conventional noncovariant notation where the time and space indices are treated distinctly. To make the transition, we note that on a Newton-Cartan background, we can choose a basis $\left\{x^{\mu}\right\}=\left\{t, x^{i}\right\}$ such that the Galilean frame velocity $\left(v^{\mu}\right)=\partial_{t}$. In this basis, we decompose the Newton-Cartan structure as

$$
\begin{aligned}
n_{\mu} & =\left(\begin{array}{l}
1 \\
0
\end{array}\right), \quad v^{\mu}=\left(\begin{array}{l}
1 \\
0
\end{array}\right), \quad p^{\mu \nu}=\left(\begin{array}{cc}
0 & 0 \\
0 & \delta^{i j}
\end{array}\right), \\
p_{\mu \nu} & =\left(\begin{array}{ll}
0 & 0 \\
0 & \delta_{i j}
\end{array}\right), \quad B_{\mu}^{(v)}=0,
\end{aligned}
$$

where $\delta^{i j}=\delta_{i j}$ is the Kronecker delta. It can be checked that the respective Newton-Cartan connection $\Gamma_{\mu \nu}^{\lambda}=0$, justifying the spacetime to be flat. The Newton-Cartan structure in the fluid frame can also be worked out from here to be

$$
\begin{aligned}
u^{\mu} & =\left(\begin{array}{c}
1 \\
u^{i}
\end{array}\right), \quad B_{\mu}=\left(\begin{array}{c}
-\frac{1}{2} u^{k} u_{k} \\
u_{i}
\end{array}\right), \\
p^{\mu \nu} & =\left(\begin{array}{cc}
0 & 0 \\
0 & \delta^{i j}
\end{array}\right), \quad p_{\mu \nu}=\left(\begin{array}{cc}
u^{k} u_{k} & -u_{j} \\
-u_{i} & \delta_{i j}
\end{array}\right) .
\end{aligned}
$$

\begin{tabular}{|c|c|}
\hline Coefficient & $t_{\mathrm{mf}}^{\mu \nu}$ \\
\hline$P$ & $P p^{\mu \nu}$ \\
\hline$\eta$ & $-\eta \sigma^{\mu \nu}$ \\
\hline$\zeta$ & $-\zeta p^{\mu \nu} \Theta$ \\
\hline$\alpha_{1}$ & $\begin{array}{c}\frac{1}{2} \alpha_{1} p^{\mu \nu} \bar{\tau}_{\varpi}^{\rho} \bar{\tau}_{\varpi \rho}-\alpha_{1} \bar{\tau}_{w}^{\mu} \bar{\tau}^{\mu \nu} \bar{\tau}_{\varpi}^{\rho} \bar{\tau}_{\varpi \rho}-\alpha_{1} \bar{\tau}_{\varpi}^{\mu} \bar{\tau}_{\varpi}^{\nu}-p^{\mu \nu} \frac{\partial P}{\partial E} \frac{1}{2} \frac{\partial\left(T \alpha_{1}\right)}{\partial T} \bar{\tau}_{\varpi}^{\rho} \bar{\tau}_{\varpi \rho} \\
-p^{\mu \nu} \frac{\partial P}{\partial R} \frac{1}{2}\left(\frac{1}{2} \frac{\partial \alpha_{1}}{\partial \varpi} \bar{\tau}_{\varpi}^{\mu} \bar{\tau}_{\varpi \mu}-\tilde{\nabla}_{\nu}\left(\alpha_{1} T \bar{\tau}_{\varpi}^{\nu}\right)\right)\end{array}$ \\
\hline$\alpha_{2}$ & $\begin{array}{c}\left.p^{\mu \nu} \alpha_{2} \bar{\tau}_{\varpi}^{\rho} \bar{\tau}_{\rho}-2 \alpha_{2} \bar{\tau}_{\varpi}^{(\mu} \bar{\tau}^{\nu)} \quad p^{\mu \nu} \alpha_{2} \bar{\tau}_{\varpi}^{\rho} \bar{\tau}_{\rho}-2 \alpha_{2} \bar{\tau}_{\varpi}^{(\mu} \bar{\tau}^{\nu}\right) \\
-p^{\mu \nu} \frac{\partial P}{\partial E}\left(T^{2} \frac{\partial\left(\alpha_{2} / T\right)}{\partial T} \bar{\tau}_{\varpi}^{\rho} \bar{\tau}_{\rho}-T \nabla_{\nu}\left(\frac{\alpha_{2}}{T} \bar{\tau}_{\varpi}^{\nu}\right)\right) \\
-p^{\mu \nu} \frac{\partial P}{\partial R}\left(\frac{\partial \alpha_{2}}{\partial \varpi} \bar{\tau}_{\varpi}^{\rho} \bar{\tau}_{\rho}-\tilde{\nabla}_{\nu}\left(\alpha_{2} T \bar{\tau}^{\nu}\right)\right)\end{array}$ \\
\hline$\alpha_{3}$ & $\begin{array}{c}\frac{1}{4} \alpha_{3} p^{\mu \nu} \omega^{\rho \sigma} \omega_{\rho \sigma}^{\mu \nu} \omega^{\rho \sigma} \omega_{\rho \sigma} \omega_{\rho}^{\mu}{ }_{\rho} \omega^{\nu \rho} \alpha_{3} \omega^{\mu} \omega^{\nu \rho} \\
-p^{\mu \nu} \frac{\partial P}{\partial E} \frac{T^{2}}{4} \frac{\partial\left(\alpha_{3} / T\right)}{\partial T} \omega^{\nu \rho} \omega_{\nu \rho}-p^{\mu \nu} \frac{\partial P}{\partial R} \frac{1}{4 T} \frac{\partial \alpha_{3}}{\partial T} \omega^{\rho \sigma} \omega_{\rho \sigma}\end{array}$ \\
\hline$\alpha_{5}$ & $\begin{array}{c}p^{\mu \nu}\left(\alpha_{5} R-2 p^{\rho \sigma} \tilde{\nabla}_{\rho} \tilde{\nabla}_{\sigma} \alpha_{5}\right)-2 p^{\mu \rho} p^{\nu \sigma}\left(\alpha_{5} R_{\rho \sigma}-\tilde{\nabla}_{\rho} \tilde{\nabla}_{\sigma} \alpha_{5}\right) \quad p^{\mu \nu}\left(\alpha_{5} R-2 p^{\rho \sigma} \tilde{\nabla}_{\rho} \tilde{\nabla}_{\sigma} \alpha_{5}\right)-2 p^{\mu \rho} p^{\nu \sigma}\left(\alpha_{5} R_{\rho \sigma}-\tilde{\nabla}_{\rho} \tilde{\nabla}_{\sigma} \alpha_{5}\right) \\
-p^{\mu \nu} \frac{\partial P}{\partial E}\left(T^{2} \frac{\partial\left(\alpha_{5} / T\right)}{\partial T} R+2 p^{\rho \sigma} \tilde{\nabla}_{\rho} \tilde{\nabla}_{\sigma} \alpha_{5}\right) \\
-p^{\mu \nu} \frac{\partial P}{\partial R} \frac{\partial \alpha_{5}}{\partial \varpi} R\end{array}$ \\
\hline$\delta_{1}^{-}$ & $-\delta_{1}^{+} \tau^{\langle\mu} \tau^{\nu\rangle}$ \\
\hline$\delta_{2}^{-}$ & $-\delta_{2}^{-} p^{\mu \nu} \tau^{\rho} \tau_{\rho}$ \\
\hline$\delta_{3}^{+}$ & $-2 \delta_{3}^{+} \sigma^{\mu \nu} \Theta$ \\
\hline$\delta_{3}^{-}$ & $-\delta_{3}^{-} p^{\mu \nu} \sigma^{\rho \sigma} \sigma_{\rho \sigma}$ \\
\hline$\delta_{4}$ & $-\delta_{4} \sigma^{\langle\mu \rho} \sigma_{\rho}^{\nu\rangle}$ \\
\hline$\delta_{5}$ & $-2 \delta_{5} p^{\mu \nu} \Theta^{2}$ \\
\hline$\delta_{6}^{-}$ & $-\delta_{6}^{+} \bar{\tau}_{\varpi}^{\langle\mu} \tau^{\mu\rangle}$ \\
\hline$\delta_{\overline{7}}^{-}$ & $-\delta_{\overline{7}}^{-} p^{\mu \nu} \bar{\tau}_{\varpi}^{\rho} \tau_{\rho}$ \\
\hline $\bar{\delta}_{9}$ & $-\bar{\delta}_{9} \omega^{\langle\mu \rho} \sigma_{\rho}{ }^{\nu\rangle}$ \\
\hline$\delta_{10}^{-}$ & $-p^{\mu \nu}\left[\delta_{10}^{-} \tilde{\nabla}_{\rho} \tau^{\rho}+\frac{T}{2} \tau^{\rho} \partial_{\rho}\left(\frac{\delta_{10}^{-}}{T}\right)\right]$ \\
\hline$\delta_{11}^{-}$ & $-P^{\rho\langle\mu} P_{\sigma}^{\nu\rangle}\left[\delta_{11}^{-} \tilde{\nabla}_{\rho} \tau^{\sigma}+\frac{T}{2} \tau^{R} \partial_{T}\left(\frac{\delta_{11}^{-1}}{T}\right)\right]$ \\
\hline $\bar{\delta}_{12}$ & $-2 p^{\mu \nu}\left[\bar{\delta}_{12} u^{\rho} \partial_{\rho} \Theta+\frac{T}{2} \Theta u^{\rho} \partial_{\rho}\left(\frac{\bar{\delta}_{12}}{T}\right)\right]-p^{\mu \nu} \bar{\delta}_{12}\left(\Theta^{2}-a^{\rho} \tau_{\rho}\right)$ \\
\hline $\bar{\delta}_{13}$ & $-\left[\bar{\delta}_{13} u^{\rho} \tilde{\nabla}_{\rho} \sigma^{\mu \nu}+\frac{T}{2} \sigma^{\mu \nu} u^{\rho} \partial_{\rho}\left(\frac{\bar{\delta}_{13}}{T}\right)\right]-\bar{\delta}_{13}\left(\frac{1}{2} \sigma^{\mu \nu} \Theta-a^{\langle\mu} \tau^{\nu\rangle}\right)$ \\
\hline
\end{tabular}

TABLE X. Stress-energy tensor of a Galilean fluid up to second derivative order in offshell frame and mass frame respectively. Note that the only difference in the two frames comes in the hydrostatic sector. 
We have enlisted the one derivative fluid data in Table VII to aid the transition of the constitutive relations to noncovariant notation.

In flat spacetime, the conservation laws take the wellknown form

$$
\begin{gathered}
\text { Mass Conservation: } \partial_{t} \rho^{t}+\partial_{i} \rho^{i}=0 \\
\text { Energy Conservation: } \partial_{t} \epsilon_{(v)}^{t}+\partial_{i} \epsilon_{(v)}^{i}=0
\end{gathered}
$$$$
\text { Momentum Conservation: } \partial_{t} \rho^{j}+\partial_{i} t_{(v)}^{i j}=0 \text {. }
$$

Here we have identified various Galilean quantities as expressed in the Galilean frame defined by $\partial_{t}$ : mass density $\rho^{t}$, mass current $\rho^{i}$, energy density $\epsilon_{(v)}^{t}$, energy current $\epsilon_{(v)}^{i}$, and stress tensor $t_{(v)}^{i j}$. For a Galilean fluid, they take a schematic form

$$
\begin{aligned}
\rho^{t} & =R+\varsigma_{\rho}, \quad \rho^{i}=\rho^{t} u^{i}+\varsigma_{\rho}^{i}, \\
t_{(v)}^{i j} & =P \delta^{i j}+\rho^{t} u^{i} u^{j}+2 u^{(i} \varsigma_{\rho}^{j)}+\varsigma_{t}^{i j}, \\
\epsilon_{(v)}^{t} & =E+\frac{1}{2}\left(R+\varsigma_{\rho}\right) u^{k} u_{k}+\varsigma_{\rho}^{k} u_{k}+\varsigma_{\epsilon}, \\
\epsilon_{(v)}^{i} & =\left(\epsilon_{(v)}^{t}+P\right) u^{i}+\frac{1}{2} \varsigma_{\rho}^{i} u^{k} \bar{u}_{k}+\varsigma_{\epsilon}^{i}+\varsigma_{t}^{i j} u_{j},
\end{aligned}
$$

where $\varsigma_{\rho}, \varsigma_{\rho}^{i}, \varsigma_{\epsilon}, \varsigma_{\epsilon}^{i}$, and $\varsigma_{t}^{i j}$ contain all the derivative corrections. We can also use the mass hydrodynamic frame

$$
\begin{aligned}
\rho_{\mathrm{mf}}^{t} & =R, \quad \rho_{\mathrm{mf}}^{i}=R u^{i}, \\
t_{(v), \mathrm{mf}}^{i j} & =P \delta^{i j}+R u^{i} u^{j}+\varsigma_{t, \mathrm{mf}}^{i j}, \\
\epsilon_{(v), \mathrm{mf}}^{t} & =E+\frac{1}{2} R u^{k} u_{k}, \\
\epsilon_{(v), \mathrm{mf}}^{i} & =\left(E+P+\frac{1}{2} R u^{k} u_{k}\right) u^{i}+\varsigma_{\epsilon, \mathrm{mf}}^{i}+\varsigma_{t, \mathrm{mf}}^{i j} u_{j} .
\end{aligned}
$$

in which the expressions look most familiar. For the constitutive relations up to second order, the respective derivative corrections can be directly read out from Tables VIII-X using results in Table VII.

We have now completed the most generic analysis of the constitutive relations of a Galilean fluid up to second derivative order. Before closing this paper, in the next section we present an example of how these second order terms might find relevance in a physical process. We consider a ball being dragged through a Galilean fluid and study corrections to the Stokes' law due to a representative second order term.

\section{SECOND ORDER CORRECTIONS TO THE STOKES' LAW}

In this section, we analyse the effect of the second order transport coefficients on a well known hydrodynamic phenomenon, namely the Stokes' law [29]. The physical setup is the following: we are interested in finding out the fluid profile around a spherical ball that is moving at a constant velocity in a fluid of infinite extent. Given the symmetries of the problem, we will choose a spherical coordinate system $(r, \theta, \phi)$. The radius of the ball $\ell$, its constant velocity $U \hat{z}$ with respect to the fluid at infinity, fluid density $R$, and fluid viscosity $\eta$ are the parameters of the problem. The fluid resists the motion of the ball due to its viscosity by applying a drag force opposite to its direction of motion. Its magnitude is determined by the Stokes' law

$$
\vec{F}=-6 \pi \eta \ell U \hat{z}
$$

This law follows from the momentum conservation Eq. (75), commonly known as the Navier-Stokes (NS) equation, under certain assumptions which we outline below. For a first order dissipative nonrelativistic fluid NS equation takes the form

$R \partial_{t} u^{i}+R u^{j} \partial_{j} u^{i}=-\partial^{i} P+\eta \partial^{2} u^{i}+\left(\zeta+\frac{1}{3} \eta\right) \partial^{i} \partial_{j} u^{j}$,

where the transport coefficients $\eta, \zeta$ have been assumed to be constant in spacetime. The problem can be made time independent by working in the rest frame of the ball, so that far away from the ball, the velocity of fluid is $-U \hat{z}$. We will ignore any inertial effects all together. Next we consider the fluid to be incompressible ( $R$ is constant) and hence by virtue of the equation of continuity (mass conservation equation), the fluid velocity becomes divergenceless

$$
\partial_{t} R+\partial_{i}\left(R u^{i}\right)=R \partial_{i} u^{i}=0 .
$$

These assumptions simplify the NS equation Eq. (79) to be

$$
R u^{j} \partial_{j} u^{i}=-\partial^{i} P+\eta \partial^{2} u^{i} .
$$

Due to the axisymmetric nature of our problem, $u^{\phi}$ can be taken to be 0 and all the other fields to be independent of the $\phi$ coordinate. With this ansatz, the fluid velocity can be expressed in terms of a stream function $\psi(r, \theta)$

$$
\vec{u}=\frac{1}{r^{2} \sin \theta}\left(\partial_{\theta} \psi \hat{r}-\partial_{r} \psi \hat{\theta}\right)
$$

So, for a given fluid (characterized by $\rho, \eta$ ), the problem is reduced to solving Eq. (81) for the pressure $P$ and the stream function $\psi$, with boundary conditions: (1) $\mathrm{P}=P_{0}$ is constant at infinity, (2) $\vec{u}=-U \hat{z}$ at infinity, and (3) $\vec{u}=0$ at the surface of the ball. The solutions are given as 


$$
\begin{aligned}
\psi & =\frac{1}{2} U \sin ^{2} \theta\left(\frac{\ell^{3}}{2 r}-\frac{3}{2} \ell r+r^{2}\right), \\
P & =P_{0}-\eta \ell \frac{3 U \cos \theta}{2 r^{2}}
\end{aligned}
$$

We define the force per unit area on the ball as

$$
\begin{aligned}
\mathrm{d} \vec{F} & =t^{i j} \hat{x}_{i} \mathrm{~d} a_{j}=\ell^{2} \sin \theta \mathrm{d} \phi \mathrm{d} \theta\left(P \hat{r}-\eta \sigma^{r \theta} \hat{\theta}\right) \\
& =\ell^{2} \sin \theta \mathrm{d} \phi \mathrm{d} \theta\left(P_{0} \hat{r}-\frac{3 \eta U}{2 \ell} \hat{z}\right) .
\end{aligned}
$$

Stokes' law follows from here by integrating this equation over the surface of the ball.

\section{A. Navier-Stokes equation with second order scalar corrections}

We now study the Navier-Stoke equation equation (79) in presence of second order transport coefficients. In particular, we want to see the second order corrections to the Stokes' law [30] given in Eq. (78). The full analysis of Eq. (79) in presence of all second order coefficients is naturally very involved, and is out of the scope of this paper. We plan to return to this analysis in the future. Here, we are only interested in the second order scalar terms. As it turns out, such terms do not change the fluid velocity profile and only affect the pressure.

We modify the stress tensor of our nonrelativistic fluid with a scalar term proportional to a second order nonhydrostatic data $S$, giving us

$$
t^{i j}=R u^{i} u^{j}+P \delta^{i j}-\eta \sigma^{i j}+\tilde{\eta} S \delta^{i j},
$$

leaving the energy and mass currents unmodified. $\tilde{\eta}$ is the associated transport coefficient. In terms of the transport coefficients defined in Table X, $\tilde{\eta}$ can be a linear combination of [31]

$\delta_{2}^{-}, \quad \delta_{3}^{-}, \quad \delta_{5}, \quad \delta_{7}^{-}$,

$\delta_{10}^{-}-\frac{1}{2} \delta_{1}^{+}-\frac{1}{2 d} \delta_{2}^{+}+\frac{1}{2} \bar{\delta}_{8}, \quad \bar{\delta}_{12}+\frac{E+P}{R} \delta_{2}^{+}+\delta_{7}^{+}$.

Under the assumptions of incompressibility and constant transport coefficients, the associated NS equation takes the form

$$
\partial^{i} P+R u^{j} \partial_{j} u^{i}-\eta \partial^{2} u^{i}+\tilde{\eta} \partial^{i} S=0 .
$$

Taking a curl of this equation, $P, S$, and $\tilde{\eta}$ drop out of the equation. The resultant equation is just an equation in the velocity ignorant of our $S$ corrections. Together with the divergence-less condition, it completely determines the velocity profile which is independent of $S$ and is simply given by Eq. (82).
Once we have obtained the velocity profile, the pressure $P$ can be obtained by solving Eq. (87). In general, the solutions will crucially depend on the form of $S$. For example, if $S$ only involves the velocity and its derivatives, Eq. (87) simply becomes a homogeneous first order differential equation for $P$. The solution is given by a trivial extension of Eq. (83)

$$
P=P_{0}-\eta \ell \frac{3 U \cos \theta}{2 r^{2}}-\tilde{\eta} S,
$$

where $S$ is evaluated on the velocity profile. Since the stress tensor in Eq. (85) only depends on the combination $P+\tilde{\eta} S$, we can see that the contributions from $\tilde{\eta}$ drop out of it after plugging in the solutions. It follows from Eq. (84) therefore, that the Stokes' law does not receive any corrections. In fact the same argument goes through for any arbitrary $S$. Note that Eq. (87) can be rewritten as

$$
\partial^{i}(P+\tilde{\eta} S)=-R u^{j} \partial_{j} u^{i}+\eta \partial^{2} u^{i} .
$$

After plugging in the solution for velocity, this equation can be integrated once to give

$$
P+\tilde{\eta} S=P_{0}-\eta \ell \frac{3 U \cos \theta}{2 r^{2}} .
$$

Depending on the pressure dependence of $S$, this equation might be nontrivial to solve. However, as far as $t^{i j}$ is concerned, we are only interested in the combination $P+\tilde{\eta} S$. It follows therefore, that $t^{i j}$ and hence the Stokes' law does not receive any corrections due to $\tilde{\eta}$.

We have argued that the nonhydrostatic second order scalar corrections $S$ do not affect the Stokes' law under the assumptions of incompressibility and constant transport coefficients (which are the same as imposed by Landau in his book [32]). To see a nontrivial effect on the Stokes' law therefore, we should consider other tensorial corrections to the stress tensor. The full analysis however, is expected to be pretty involved analytically, and we intend to return to this in the near future.

\section{DISCUSSION}

In this paper, we have performed a complete second order analysis of uncharged parity-even nonrelativistic hydrodynamics using null fluid formalism. Second order terms in relativistic hydrodynamics are required to maintain causality. If we look at our theory as a nonrelativistic limit of a relativistic theory, we might expect to see some signatures of the causality requirement [33]. It is therefore important to study the effect of second order terms in nonrelativistic fluid constitutive relations. The respective results can be found in Sec. IV B in Tables VIII-X. To summarize, there are 25 new transport coefficients that appear at second order. 5 of them are hydrostatic, i.e., they 
determine the equilibrium configuration of the fluid. Nine others are dissipative, i.e., they are responsible for the production of entropy during dynamical processes, while the remaining 11 quantify dynamical processes which do not cause dissipation.

One might question if the analysis we have performed indeed reproduces the most generic second-order Galilean fluid, or if there are any inherent constraints arising due to the framework of null reduction. Indeed, as we have argued in our original paper on null fluids [11], a $(d+2)$ dimensional null fluid is in exact correspondence with a $(d+1)$-dimensional Galilean fluid. This claim is further bolstered by the work of [34] which showed that a Galilean fluid can be covariantly arranged into a one higher dimensional system, which is essentially a null fluid [12]. In fact, all the analysis we have done here could also be done directly in a $(d+1)$-dimensional Newton-Cartan framework of Galilean hydrodynamics. As all the Galilean symmetries are manifest in form of Poincaré symmetries in null fluid framework, the analysis becomes much more natural and robust.

To understand the physical effect of the new second order terms, we have also explored how some of these might modify the well known Stokes' law, which tells us the drag force experienced by a body while moving through a fluid. To first order, we already know that it is proportional to the shear viscosity of the fluid [32]. We concluded that nonhydrostatic scalar corrections to the stress tensor (terms that appear in the stress tensor as $t^{i j} \sim \tilde{\eta} S \delta^{i j}$ ) do not affect the Stokes' law at all. This accounts for 6 out of 25 transport coefficients. There are 7 other terms which only affect the energy profile and hence cannot contribute to the drag force. The remaining 12 coefficients can in principle however, affect the Stokes' law in a nontrivial manner. One particular term of interest would be the so called "relaxation" coupling to $\left(\partial_{t}+u^{k} \partial_{k}\right) \sigma^{i j}$ in the stress tensor, analogue of which was required in relativistic fluids to salvage causality. Muller, Israel, and Stewart noted in [4-6] that the causal structure of relativistic hydrodynamics can be recovered by adding a second order term proportional to $u^{\rho} \nabla_{\rho} \sigma^{\mu \nu}$ to the energy-momentum tensor. The associated transport coefficient is known as the "relaxation time."
For a nonrelativistic fluid, the corresponding term is related to $\bar{\delta}_{13}$ in Table X. It will be interesting to study the effect of this term on the Stokes' law. The analysis however, is quite involved. We intend to come back to this study in a future project.

Another prospective direction would be to compute the explicit form of these second order transport coefficients using holography. The principles of hydrodynamics and the second law of thermodynamics allow us to pen down the constitutive relations of a fluid up to some unknown transport coefficients. Details of these transport coefficients, depend on the particular fluid in question and the details of the microscopic theory. Holography however, allows us to compute these coefficients directly for a particular class of fluids. For relativistic fluids, using the fluid/gravity correspondence [35,36], transport coefficients for a holographic plasma has been successfully computed (see [35-43] and references therein). For a first order nonrelativistic fluid, some progress in this direction has been made in [14]. We would like to set up a holographic model dual to our null fluid construction and use it to compute the associated transport coefficients. It would be interesting to see what this analysis has to tell us about the second order transport coefficients talked about in this paper.

\section{ACKNOWLEDGMENTS}

We would like to thank Suvankar Dutta for various useful discussions and collaboration during the initial stages of this work. We would also like to thank Jyotirmoy Bhattacharya and Ruth Gregory for helpful comments during the course of this project. We are thankful to IISER Bhopal where this project was initiated. A. J. would like to thank the hospitality at IISER Pune and Perimeter Institute where part of this work was done. Work of N. B. is partially supported by a DST/SERB Ramanujan Fellowship. A. J. is supported by the Durham Doctoral Scholarship offered by Durham University. S. B. is thankful to Infosys Foundation for a travel grant. Finally, we thank the people of India for their generous support for the basic sciences.
[1] W. A. Hiscock and L. Lindblom, Phys. Rev. D 35, 3723 (1987).

[2] W. A. Hiscock, Phys. Rev. D 33, 1527 (1986).

[3] W. A. Hiscock and L. Lindblom, Ann. Phys. (N.Y.) 151, 466 (1983).

[4] W. Israel and J. M. Stewart, Ann. Phys. (N.Y.) 118, 341 (1979).

[5] W. Israel, Ann. Phys. (N.Y.) 100, 310 (1976).
[6] I. Muller, Z. Phys. 198, 329 (1967).

[7] H. Song and U.W. Heinz, J. Phys. G 36, 064033 (2009).

[8] M. Luzum and P. Romatschke, Phys. Rev. C 78, 034915 (2008); 79, 039903 (2009).

[9] S. Bhattacharyya, J. High Energy Phys. 07 (2012) 104.

[10] N. Banerjee, S. Dutta, and A. Jain, Phys. Rev. D 92, 081701 (2015). 
[11] N. Banerjee, S. Dutta, and A. Jain, Phys. Rev. D 93, 105020 (2016).

[12] A. Jain, Phys. Rev. D 93, 065007 (2016).

[13] N. Banerjee, S. Dutta, and A. Jain, Phys. Rev. D 96, 065004 (2017).

[14] M. Rangamani, S. F. Ross, D. T. Son, and E. G. Thompson, J. High Energy Phys. 01 (2009) 075.

[15] N. Banerjee, J. Bhattacharya, S. Bhattacharyya, S. Jain, S. Minwalla, and T. Sharma, J. High Energy Phys. 09 (2012) 046.

[16] K. Jensen, M. Kaminski, P. Kovtun, R. Meyer, A. Ritz, and A. Yarom, Phys. Rev. Lett. 109, 101601 (2012).

[17] R. Loganayagam, arXiv:1106.0277.

[18] D. T. Son and P. Surowka, Phys. Rev. Lett. 103, 191601 (2009).

[19] K. Jensen, R. Loganayagam, and A. Yarom, J. High Energy Phys. 05 (2014) 134.

[20] J. Bhattacharya, S. Bhattacharyya, S. Minwalla, and A. Yarom, J. High Energy Phys. 05 (2014) 147.

[21] S. Bhattacharyya, S. Jain, S. Minwalla, and T. Sharma, J. High Energy Phys. 01 (2013) 040.

[22] A. Jain, Phys. Rev. D 95, 121701 (2017).

[23] J. Armas, J. Bhattacharya, A. Jain, and N. Kundu, J. High Energy Phys. 06 (2017) 090.

[24] F. M. Haehl, R. Loganayagam, and M. Rangamani, Phys. Rev. Lett. 114, 201601 (2015).

[25] F. M. Haehl, R. Loganayagam, and M. Rangamani, J. High Energy Phys. 05 (2015) 060.

[26] S. Bhattacharyya, J. High Energy Phys. 08 (2014) 165.

[27] S. Bhattacharyya, J. High Energy Phys. 07 (2014) 139.

[28] K. Jensen, arXiv:1408.6855.
[29] This is a very famous problem and described in many texts on fluid dynamics.

[30] We thank Suvankar Dutta for suggesting this application.

[31] The combinations of transport coefficients are specifically chosen, so that the energy current does not get any second order corrections.

[32] L. Landau and E. Lifshitz, Fluid Mechanics, Teoreticheskaia fizika (Pergamon Press, New York, 1959).

[33] A direct notion of causality in nonrelativistic systems would be related to a preferred time-slicing. In this setting, causality will prevent propagation of any signal on a given time slice to an earlier time slice. We thank the referee for clarifying this point.

[34] M. Geracie, K. Prabhu, and M. M. Roberts, J. High Energy Phys. 08 (2015) 042.

[35] S. Bhattacharyya, V.E. Hubeny, S. Minwalla, and M. Rangamani, J. High Energy Phys. 02 (2008) 045.

[36] R. Baier, P. Romatschke, D. T. Son, A. O. Starinets, and M. A. Stephanov, J. High Energy Phys. 04 (2008) 100.

[37] G. Policastro, D. T. Son, and A. O. Starinets, Phys. Rev. Lett. 87, 081601 (2001).

[38] N. Banerjee and S. Dutta, Nucl. Phys. B845, 165 (2011).

[39] D. Astefanesei, N. Banerjee, and S. Dutta, J. High Energy Phys. 02 (2011) 021.

[40] N. Banerjee and S. Dutta, J. High Energy Phys. 08 (2010) 041.

[41] J. Erdmenger, M. Haack, M. Kaminski, and A. Yarom, J. High Energy Phys. 01 (2009) 055.

[42] N. Banerjee, J. Bhattacharya, S. Bhattacharyya, S. Dutta, R. Loganayagam, and P. Surowka, J. High Energy Phys. 01 (2011) 094.

[43] S. Dutta, J. High Energy Phys. 05 (2008) 082. 